\title{
Automorfismos De GRAFos
}

Eduardo Tengan

\author{
DISSERTAÇÃO APRESENTADA \\ $A O$ \\ INSTITUTO DE MATEMÁTICA E ESTATÍSTICA \\ DA \\ UNIVERSIDADE DE SÃO PAULO \\ PARA \\ OBTENÇÃO DO GRAU DE MESTRE \\ EM \\ CIÊNCIA DA COMPUTAÇÃO
}

Área de Concentração: Combinatória

Orientador: Prof. Dr. Arnaldo Mandel

São Paulo, 22 de maio de 2002 


\section{Automorfismos DE GRAFOS}

Este exemplar corresponde à redação final da dissertação, devidamente corrigida e defendida por Eduardo Tengan, aprovada pela comissão julgadora.

São Paulo, 22 de maio de 2002

Banca Examinadora:

- Prof. Dr. Arnaldo Mandel, IME/USP (orientador)

- Prof. Dra. Célia Picinin de Mello, IC/UNICAMP

- Prof. Dr. Yoshiharu Kohayakawa, IME/USP 


\subsection{Resumo}

Este trabalho descreve alguns métodos algébricos para o cálculo de grupos de automorfismos de grafos. Dois métodos são enfocados: a utilização da teoria de grupos de permutações, base do algoritmo de Luks, que determina, em tempo polinomial, o grupo de automorfismos de um grafo de grau limitado; e uma reformulação do problema em termos de extensões de corpos, que procura utilizar especializações para obter informações sobre grupos de automorfismos de grafos.

\subsection{Abstract}

This work describes some algebraic methods for the determination of automorphism groups of graphs. Two methods are focused: the use of the permutation groups, central to Luks' algorithm, which determines in polinomial time the automorphism group of a graph of bounded degree; and a reformulation of the problem in terms of field extensions that tries to use specializations in order to get information about automorphism group of graphs. 


\section{$0.3 \quad$ Agradecimentos}

Este trabalho não teria sido concretizado sem o apoio de algumas pessoas, as quais gostaria de agradecer neste momento.

A lista desta pessoas que direta ou indiretamente contribuiram para este trabalho é demasiada longa para que eu a inclua neste pequeno espaço. Como representantes desta enorme lista, cito algumas pessoas, às quais devo especial reconhecimento.

Em primeiro lugar, o meu orientador, Arnaldo Mandel, pela paciência, pela orientação e pelo empenho. Sua grande erudição e seu humor ímpar tornaram especialmente interessantes e agradáveis as várias horas de discussões que resultaram nesta monografia.

Também gostaria de agradecer o professor Yoshiharu Kohayakawa por sua ajuda e seu constante interesse. Quer seja pelas sugestões, quer seja pelos cursos, quer seja pelas referências, sua influência permeia este trabalho.

Devo ainda à professora Yoshiko Wakabayashi meus sinceros agradecimentos por sua orientação e dedicação. Espero ter feito bom uso dos conhecimentos a mim confiados em suas preleções.

Finalmente, quero deixar claro que nenhuma destas pessoas é responsável pelos erros (poucos, assim espero) que aqui restaram. Eles são, naturalmente, inteira responsabilidade minha e do acaso. 
"To get a book from these texts, only scissors and glue were needed... Strangely enough, the different pieces fitted well together."

J.-P. Serre, comentando sobre a origem do livro "A Course in Arithmetic" ao receber o Steele Prize 


\section{Sumário}

0.1 Resumo .......................... 2

0.2 Abstract . . . . . . . . . . . . . . . 2

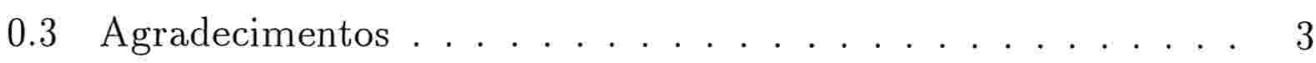

1 Introdução $\quad 7$

1.1 Isomorfismos e Automorfismos de Grafos . . . . . . . . . . . 7

1.2 Outros Problemas Computacionais . . . . . . . . . . . . . 9

1.3 Mais questões sobre automorfismos . . . . . . . . . . . . . 11

1.4 Resumo dos demais capítulos . . . . . . . . . . . . . 12

2 Grupos de Permutações e Algoritmos $\quad 15$

2.1 Grupos de Permutações . . . . . . . . . . . . . . . 15

2.2 Grupos Transitivos . . . . . . . . . . . . . . . . . . . . . . . . . . . . 17

2.3 Cadeias de Estabilizadores . . . . . . . . . . . . . . 18

2.4 Subgrupos de Sylow . . . . . . . . . . . . . . . . . . . . . . . . . . . . . . . 22

2.5 Grupos Primitivos . . . . . . . . . . . . . . . . . 24

2.6 Representações Infiéis . . . . . . . . . . . . . . . . . . . 26

$\begin{array}{lll}3 & \text { O algoritmo de Luks } & 29\end{array}$

3.1 Reduzindo o problema . . . . . . . . . . . . . . . . . . . . 29

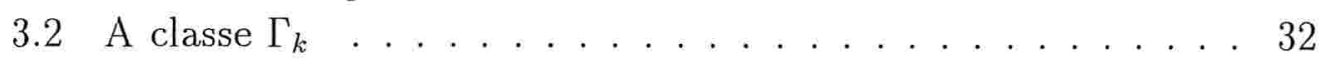

3.3 Primitividade . . . . . . . . . . . . . . . . . . . . . 34

3.4 O algoritmo de Luks . . . . . . . . . . . . . . . . . . . . . . . . . . . . . . . . . . . . 35

3.5 Socle . . . . . . . . . . . . . . . . . . . . 38

3.6 Demonstrando o resultado fundamental . . . . . . . . . . . . 43

3.6.1 O socle é abeliano . . . . . . . . . . . . . . . . . . . . . . . . . . . 43

3.6 .2 O socle não é abeliano . . . . . . . . . . . 45 
4 Automorfismos e Especializações $\quad 49$

4.1 Reformulando o problema . . . . . . . . . . . . . . 49

4.2 Um exemplo . . . . . . . . . . . . . . . . . . . . 51

4.3 Demonstrando o teorema principal . . . . . . . . . . 52

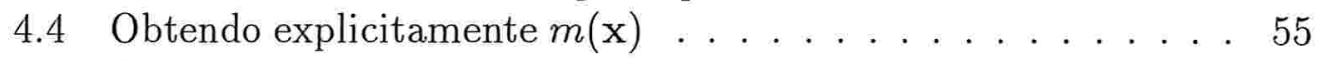

4.5 Escolhendo especializações . . . . . . . . . . . . . 58

A Notações 61

A.1 Geral . . . . . . . . . . . . . . . . . . 6 61

A.2 Grafos . . . . . . . . . . . . . . . . 61

A.3 Grupos . . . . . . . . . . . . . . . . . 61

A.4 Corpos e polinômios . . . . . . . . . . . . . . 62

A.5 Anéis e localização . . . . . . . . . . . . . . . . . 62

B Teoria de Galois $\quad 63$

B.1 Polinômios simétricos . . . . . . . . . . . . . . 63

B.2 Imersões . . . . . . . . . . . . . . . . . . . . 65

B.3 Extensões Simples. . . . . . . . . . . . . . . 67

B.4 Automorfismos e Extensões Galoisiana . . . . . . . . . . . 68

B.5 Teorema Fundamental . . . . . . . . . . . . . . . . . . 69 


\section{Capítulo 1}

\section{Introdução}

\subsection{Isomorfismos e Automorfismos de Grafos}

Dois grafos $X$ e $Y$ são isomorfos se diferem apenas na nomenclatura de seus vértices, ou seja, se existe uma bijeção $\imath: V(X) \rightarrow V(Y)$ tal que $\imath(v) \imath(w)$ é aresta de $Y$ se, e somente se, $v w$ é aresta de $X$.

Reconhecer eficientemente se dois grafos são isomorfos é um problema computacional de interesse tanto prático como teórico. Esta monografia é dedicada ao estudo deste e de outros problemas correlatos utilizando ferramentas algébricas, mais especificamente, resultados de teoria dos grupos e de teoria de Galois. Como de praxe, por um algoritmo "eficiente" entendemos um de complexidade polinomial.

A conexão deste problema com a teoria de grupos é evidenciada quando consideramos os automorfismos de um grafo $X$, isto é, os isomorfismos entre $X$ e ele mesmo. Em outras palavras, um automorfismo é uma permutação de vértices que preserva a relação de adjacência bem como a de não adjacência. Como compondo automorfismos de $X$ obtemos novamente um automorfismo de $X$, o conjunto de todos os automorfismos de $X$ é um grupo, que será denotado por $\operatorname{Aut}(X)$.

Dado um grafo $X$, há várias perguntas que podemos fazer sobre $\operatorname{Aut}(X)$. Em primeiro lugar, é possível determiná-lo explicitamente de modo eficiente?

Aqui, um cuidado: como $\operatorname{Aut}(X)$ pode ser muito grande (da ordem de $n$ !, em que $n$ é o número de vértices de $X$ ), não podemos simplesmente listar os elementos de $\operatorname{Aut}(X)$. Veremos, entretanto, que $\operatorname{Aut}(X)$ pode sempre ser escrito utilizando até $O\left(n^{2}\right)$ geradores, de modo que uma pergunta mais ra- 
zoável seria: é possível determinar geradores para $\operatorname{Aut}(X)$ de modo eficiente?

Na verdade, este último problema, o de determinar geradores para $\operatorname{Aut}(X)$, é polinomialmente equivalente a testar isomorfismo de grafos. Isto quer dizer que se dispusermos de um algoritmo eficiente para resolver um destes problemas, então é possível utilizá-lo para resolver o outro, também de maneira. eficiente. Assim, perguntamos: existe um algoritmo polinomial para resolver um destes dois problemas?

Como usual em teoria de complexidade de algoritmos, esta é mais uma daqueles perguntas razoáveis que ninguém sabe responder. Claramente, testar isomorfismo de grafos está em NP, já que um candidato a isomorfismo pode ser verificado em tempo polinomial. Entretanto, este problema se destaca dentre os demais em teoria de complexidade pois não se sabe se ele é NP-completo. Acredita-se mesmo que ele seja um problema de complexidade intermediária entre P e NP.

Evidências teóricas para esta conjectura são descritas em [Bab], dentre as quais citamos duas. A primeira é a equivalência polinomial entre os problemas de existência e de contagem de isomorfismos de grafos: em geral, problemas de contagem são mais difíceis do que os de existência, mas não para este caso. A segunda é o improvável colapso de uma hierarquia de classes de complexidade entre P e PSPACE, caso o problema de testar isomorfismo de grafos seja NP-completo.

Sob determinadas restrições, entretanto, podemos dizer muito mais sobre a complexidade destes problemas. O capítulo III desta monografia é totalmente dedicado ao algoritmo de Luks [Luk], que testa isomorfismo de grafos em tempo polinomial desde que os graus dos vértices destes grafos sejam limitados por uma constante. O algoritmo de Luks faz extenso uso da teoria de grupos de permutações. Esta tem sido uma das técnicas mais bem sucedidas no ataque deste problema mesmo no caso geral, para o qual o melhor algoritmo conhecido é também devido a Luks e gasta tempo $e^{\sqrt{O(n \log n)}}$ para grafos com $n$ vértices.

Para algumas outras classes de grafos particulares, dispõe-se de resultados ainda melhores. Por exemplo, para grafos planares, há um algoritmo linear no número de vértices. $\mathrm{E}$ para grafos com gênero $g \geq 1$, dispõe-se de testes que gastam tempo $n^{O(g)}$. Para uma lista mais completa, referimo-nos novamente a $[\mathrm{Bab}]$.

Do ponto de vista prático, algoritmos de backtrack têm bom desempenho. O programa Nauty [McK] lista todos os automorfismos de um grafo e é uma 
das implementações mais rápidas nesta categoria.

\subsection{Outros Problemas Computacionais}

Há muitos outros problemas relacionados com o teste de isomorfismo de grafos além do cálculo do grupo de automorfismos. Antes de listá-los, vamos introduzir o conceito de isomorfismo entre grafos rotulados .

Em primeiro lugar, o que é um grafo rotulado? É simplesmente um par $(X, \lambda)$, composto por um grafo $X$ e uma função $\lambda$ de $V(X)$ em um conjunto de rótulos $L$. Dois grafos rotulados (sobre um mesmo conjunto de rótulos) $\left(X, \lambda_{1}\right)$ e $\left(Y, \lambda_{2}\right)$ são isomorfos se existe um isomorfismo $\imath$ entre $X$ e $Y$ que preserva a rotulação, isto é, tal que, para todo vértice $v$ de $X$, $\lambda_{1}(v)=\lambda_{2}(\imath(v))$.

Agora podemos aumentar nossa lista de problemas computacionais relacionados a isomorfismos de grafos. Além dos dois problemas básicos

(i) Existência de Isomorfismo de Grafos: Dados dois grafos $X$ e $Y$, decidir se eles são isomorfos.

(ii) Automorfismos de um Grafo: Dado um grafo $X$, construir um conjunto gerador para $\operatorname{Aut}(X)$.

temos também

(iii) Existência de Isomorfismo de Grafos Rotulados: Dados dois grafos rotulados $\left(X, \lambda_{1}\right)$ e $\left(Y, \lambda_{2}\right)$, decidir se eles são isomorfos.

(iv) Ordem do Grupo de Automorfismos de um Grafo: Dado um grafo $X$, determinar a ordem de $\operatorname{Aut}(X)$.

(v) Número de Isomorfismos: Dados dois grafos $X$ e $Y$, determinar o número de isomorfismos entre eles.

Do ponto de vista da teoria de complexidade, os problemas acima são todos equivalentes.

Teorema 1.1 Os problemas acima são polinomialmente equivalentes. 
Vamos mostrar um esboço das reduções polinomiais entre os problemas acima. Tais reduções são conhecidas já há bastante tempo. Para maiores detalhes, veja, por exemplo, [Hof].

Comecemos com a equivalência entre (i) e (iii). O problema da existência de isomorfismo de grafos rotulados engloba o correspondente para grafos não rotulados. Entretanto, existe uma redução polinomial deste problema aparentemente mais geral para o problema sem rótulos. Dados $\left(X, \lambda_{1}\right)$ e $\left(Y, \lambda_{2}\right)$, podemos supor, sem perda de generalidade, que eles têm o mesmo número de vértices $n$ (caso contrário, os grafos são claramente não isomorfos) e que o conjunto de rótulos que efetivamente aparecem nestes grafos é $L=$ $\{1,2, \ldots, k\}$. Agora, construímos a partir de $\left(X, \lambda_{1}\right)$ um grafo não rotulado $X^{\prime}$ adicionando a cada vértice de $\left(X, \lambda_{1}\right)$ um "pingente" correspondente ao seu rótulo: para cada $v \in V(X)$, construímos um grafo completo com $n+$ $\lambda_{1}(v)$ vértices e o ligamos a $v$ por uma única aresta. Da mesma forma, construímos $Y^{\prime}$ a partir de $\left(Y, \lambda_{2}\right)$. É fácil verificar que $X^{\prime}$ e $Y^{\prime}$ são isomorfos se, e somente se, os grafos rotulados também o são.

Com o resultado acima, fica fácil ver que os problemas de existência e construção de isomorfismos são, na verdade, equivalentes: inicialmente, verificamos se existe um isomorfismo que leva um determinado vértice $v \mathrm{em}$ $X$ em um outro $w$ em $Y$ (dê um mesmo rótulo para estes vértices e um outro rótulo comum para os demais). Repetimos este processo no máximo $n$ vezes para concluir que não há isomorfismo ou que há um levando $v \mathrm{em}$ algum vértice $w$ em $Y$. Neste último caso, escolhemos outro vértice $v^{\prime}$ em $X$ e reiteramos o procedimento, só que agora nos restringindo aos isomorfismos que levam $v$ em $w$, ou seja, definimos um mesmo rótulo para $v$ e $w$, outro rótulo para $v^{\prime}$ e o vértice $w^{\prime}$ em $Y$ sendo considerado no momento e um terceiro rótulo para todos os demais vértices de $X$ e $Y$. Continuando dessa maneira, eventualmente acabamos com uma construção de um isomorfismo entre os grafos ou com uma prova de que eles não são isomorfos.

Adaptando ligeiramente a idéia anterior, podemos concluir que o problema de contagem de automorfismos se reduz ao de existência de isomorfismos. Para isto, façamos uma cópia de $X$ e apliquemos o procedimento anterior, determinando a cada passo a existência ou não de automorfismos que levam um dado vértice $v$ em outro $w$. Em outras palavras, este procedimento determina a órbita ${ }^{1} \operatorname{Aut}(X)(v)$ de um dado vértice $v \in V(X)$.

\footnotetext{
${ }^{1}$ para detalhes sobre este e outros conceitos de grupos de permutações, veja a seção sobre grupos transitivos do capítulo II
} 
Como \# $\operatorname{Aut}(X)=\# \operatorname{Aut}(X)(v) \cdot \# \operatorname{Stab}(v)$, podemos aplicar este processo recursivamente, escolhendo um outro vértice e agora nos restringindo à ação de $\operatorname{Stab}(v)$ sobre $X$, isto é, atribuindo um rótulo comum a $v$ em $X$ e em sua cópia e um rótulo diferente aos demais vértices. O produto das cardinalidades das diversas órbitas obtidas será igual ao número de automorfismos de $X$.

Temos também que (i) se reduz a (iv). Dados dois grafos $X$ e $Y$, podemos supor que eles sejam ambos conexos ou ambos não conexos, caso contrário eles não são isomorfos. Trabalhando com os complementares de $X$ e $Y$ se necessário, podemos supor ainda que $X$ e $Y$ são conexos e fazer a união disjunta de $X$ e $Y$, obtendo o grafo $Z$. Temos que \# Aut $(Z)=\# \operatorname{Aut}(X)$. \# Aut $(Y)$ se, e somente se, $X$ e $Y$ não são isomorfos.

Até o momento, sabemos que (i), (iii) e (iv) são equivalentes. Agora observe que se dois grafos $X$ e $Y$ são isomorfos, qualquer isomorfismo entre eles é a composição de um automorfismo de $X$ com um isomorfismo fixado entre $X$ e $Y$, ou seja, ou não há isomorfismos entre $X$ e $Y$ ou o número de isomorfismos é \# $\operatorname{Aut}(X)$ (que é igual a \# $\operatorname{Aut}(Y)$ ). Assim, o problema de contagem de isomorfismos se reduz ao correspondente para automorfismos. A recíproca é imediata.

Finalmente, falta considerar (ii). No capítulo II, dedicado a algoritmos sobre grupos de permutações, veremos como utilizar a técnica de cadeia de estabilizadores para determinar geradores de $\operatorname{Aut}(X)$ conhecendo-se as órbitas dos diversos vértices de $X$. Logo (iii) implica (ii). Esta mesma técnica permitirá a construção de um algoritmo, o algoritmo de Sims, que determina a ordem de um grupo a partir de um conjunto de geradores. Desta forma, se soubermos resolver (ii), saberemos também como resolver (iv). Com isto, completamos a demonstração da equivalência polinomial dos problemas acima.

\subsection{Mais questões sobre automorfismos}

Como vimos, determinar se dois grafos são isomorfos pode ser reduzido à questão de encontrar geradores para o grupo de automorfismos de um grafo $X$. Então é natural perguntar se existem outras questões sobre $\operatorname{Aut}(X)$ que são "equivalentes" ao problema do isomorfismo.

Dois problemas neste contexto são

(vi) Trivialidade do Grupo de Automorfismos de um Grafo: dado 
um grafo $X$, decidir se $\operatorname{Aut}(X)=\{1\}$.

(vii) Problema Modular da Ordem do Grupo de Automorfismos de um Grafo: dado um grafo $X$, decidir se um dado inteiro $k>1$ divide \# $\operatorname{Aut}(X)$.

Devido ao teorema anterior, é claro que decidir a trivialidade do grupo de automorfismos de um grafo é mais fácil que decidir se dois grafos são isomorfos. Infelizmente, não se sabe se existe uma redução polinomial no sentido oposto. Em [Arv], vemos que a versão modular do problema de determinar a ordem do grupo de automorfismos de um grafo é um problema de dificuldade intermediária entre estes dois, sendo, porém, desconhecido se ele é polinomialmente equivalente a um ou a outro.

Não se sabe se o problema do isomorfismo pertence a coNP, isto é, não se sabe construir certificados de não isomorfismo verificáveis em tempo polinomial. Também não se conhecem certificados polinomiais para a trivialidade do grupo de automorfismos de um grafo. No capítulo IV, veremos uma abordagem alternativa para esta questão. Mostraremos que (vi) é equivalente a testar a trivialidade do grupo de Galois de uma certa extensão de corpos. Em seguida, esboçaremos um programa para certificar a trivialidade deste grupo. Os resultados são parciais e a viabilidade deste programa ainda não é certa.

\subsection{Resumo dos demais capítulos}

Faremos um breve resumo do conteúdo de cada um dos capítulos seguintes.

O capítulo II contém diversos algoritmos sobre grupos de permutações e é essencial para o entendimento do algoritmo de Luks.

O capítulo III é inteiramente dedicado ao algoritmo de Luks, que determina se dois grafos são isomorfos em tempo polinomial, sob a restrição de que os graus dos vértices destes grafos sejam limitados. Isto é feito reduzindo-se o problema original ao cálculo do subgrupo das permutações de um conjunto colorido que preservam cores. Este problema pode ser facilmente resolvido no caso de $p$-grupos. No caso geral, introduziremos uma nova classe de grupos, $\Gamma_{k}$, que inclui todos os grupos de automorfismos de grafos. Para grafos com vértices de graus limitados, podemos tomar $k$ fixo, o que impõe restrições nos primos que são fatores das ordens destes grupos, permitindo uma redução do 
problema a um subgrupo de Sylow, para o qual então se aplica o método mencionado acima.

O último capítulo é independente dos dois anteriores e, como visto acima, descreve um possível programa para certificar a trivialidade do grupo de automorfismos de um grafo. Para isto, reduziremos este problema ao cálculo do grupo de Galois de uma extensão de corpos de funções racionais. Em seguida, mostraremos, sob certas condições, como "especializar" esta extensão, obtendo corpos mais simples, para os quais podemos calcular o grupo de Galois associado. Para conveniência do leitor, incluímos um apêndice com os resultados relevantes da teoria de Galois, com algumas demonstrações incluídas que ilustram as técnicas usadas no capítulo. 


\section{Capítulo 2}

\section{Grupos de Permutações e Algoritmos}

Antes de iniciarmos o estudo de automorfismos de grafos, precisamos nos familiarizar com técnicas e métodos da teoria computacional de grupos. Este capítulo é uma exposição de algumas destas técnicas que estão mais diretamente ligadas com nosso problema.

Começamos com uma seção sobre grupos de permutações, indicando o papel central destes na representação de grupos em um computador. Em seguida, apresentamos o conceito de cadeia de estabilizadores, que é a base para diversos algoritmos da teoria. Discutimos ainda brevemente os conceitos de transitividade e primitividade, que serão extensamente utilizados no próximo capítulo.

\subsection{Grupos de Permutações}

Dado um conjunto $\Omega$, definimos o grupo simétrico de $\Omega$ como o grupo de todas as permutações de elementos de $\Omega$. Denotamos este grupo por $\mathfrak{S}_{\Omega}$.

Freqüentemente, $\Omega=\{1,2, \ldots, n\}$ e neste caso utilizaremos a notação $\mathfrak{S}_{n}$ para representar $\mathfrak{S}_{\Omega}$. Elementos de $\mathfrak{S}_{n}$ são normalmente escritos como produto de ciclos. Um ciclo é uma permutação $\pi \in \mathfrak{S}_{n}$ tal que existe um subconjunto $\left\{i_{1}, i_{2}, \ldots, i_{r}\right\}$ dos inteiros de 1 a $n$ para o qual $\pi\left(i_{1}\right)=i_{2}$, $\pi\left(i_{2}\right)=i_{3}, \ldots, \pi\left(i_{r-1}\right)=i_{r}, \pi\left(i_{r}\right)=i_{1}$ e $\pi(j)=j$ para os demais inteiros $j$ entre 1 e $n$. Neste caso, escrevemos $\pi=\left(i_{1}, i_{2}, \ldots, i_{r}\right)$ para denotar tal ciclo. Qualquer permutação é um produto de ciclos. Por exemplo, a permutação 
$\pi \in \mathfrak{S}_{7}$ dada por

$$
\begin{array}{llll}
\pi(1)=1 & \pi(2)=3 & \pi(3)=4 & \pi(4)=2 \\
\pi(5)=6 & \pi(6)=5 & \pi(7)=7 &
\end{array}
$$

pode ser escrita simplesmente como

$$
(2,3,4)(5,6)
$$

Um subgrupo de $\mathfrak{S}_{\Omega}$ é chamado de grupo de permutações. A cardinalidade de $\Omega$ é denominada grau do grupo. Uma representação de $G$ como grupo de permutações é um homomorfismo de $G$ em $\mathfrak{S}_{\Omega}$, para algum conjunto $\Omega$ conveniente. Quando queremos explicitar o conjunto $\Omega$, diremos que $G$ age sobre $\Omega$, permutando seus elementos. Se a representação é injetiva, diremos que ela é fiel . Dado um grupo $G$ e uma representação fiel, não faremos distinções entre $G$ e sua imagem e é nesse sentido que consideraremos $G$ também um grupo de permutações.

Podemos descrever um grupo de permutações $G \subset \mathfrak{S}_{\Omega}$ através de um conjunto de geradores $g_{1}, \ldots, g_{r} \in \mathfrak{S}_{\Omega}$. Neste caso, escrevemos $G=\left\langle g_{1}, \ldots, g_{r}\right\rangle$. Como exemplo, tomemos $D_{n}$, o grupo diedral de ordem $2 n$, que é o grupo de simetrias de um polígono regular de $n$ vértices. Temos que $D_{n}$ é o subgrupo de $\mathfrak{S}_{n}$ gerado por uma reflexão $\sigma=(2, n)(3, n-1)(4, n-2) \ldots$ e por uma rotação $\pi=(1,2, \ldots, n)$.

Dado um conjunto de geradores para um grupo de permutações $G \subset \mathfrak{S}_{\Omega}$, podemos levantar várias questões computacionais relacionadas. Por exemplo, gostaríamos de poder decidir se um determinado elemento $\pi \in \mathfrak{S}_{\Omega}$ está ou não em $G$. Outro problema seria calcular a ordem de $G$. Veremos mais adiante que estas e outras questões podem ser resolvidas de modo eficiente por algoritmos polinomiais no grau e no número de geradores de $G$.

À primeira vista, pode parecer que grupos de permutações são estruturas extremamente particulares. Na verdade, o teorema de Cayley diz exatamente o contrário: todo grupo $G$ é isomorfo a um grupo de permutações. Basta considerar a chamada representação regular de $G$ : um elemento $g \in G$ pode ser identificado com a permutação $\pi \in \mathfrak{S}_{G}$ que leva $x \in G$ em $\pi(x)=g x$.

Podemos ainda generalizar a construção anterior da seguinte maneira: seja $H$ um subgrupo de $G$ e seja $C=\left\{g_{1} H, g_{2} H, \ldots, g_{r} H\right\}$ o conjunto das classes laterais à esquerda de $H$. Considere a representação de $G$ em $\mathfrak{S}_{C}$ que associa cada elemento $g \in G$ à permutação dada por

$$
g_{i} H \rightarrow g g_{i} H, \quad \text { para } 1 \leq i \leq r
$$


Chamaremos esta representação de representação regular associada a $H$.

Qual o kernel desta representação? Note que $g g_{i} H=g_{i} H \Longleftrightarrow g g_{i} \in$ $g_{i} H \Longleftrightarrow g \in g_{i} H g_{i}^{-1}$. Então $g$ está no kernel se, e somente se, $g$ está em $g_{i} H g_{i}^{-1}$ para todo $i$. Resumimos este importante resultado no

Teorema 2.1 Seja $H$ um subgrupo de um grupo G. O kernel da representação regular associada a $H$ é o maior subgrupo normal de $G$ contido em $H$.

De fato, dado $g=g_{i} h, h \in H$, temos que $g H g^{-1}=g_{i} h H h^{-1} g_{i}^{-1}=g_{i} H g_{i}^{-1}$. Conseqüentemente, o maior subgrupo normal de $G$ contido em $H$ é

$$
\bigcap_{g \in G} g H g^{-1}=\bigcap_{1 \leq i \leq r} g_{i} H g_{i}^{-1}
$$

Representações de grupos como grupo de permutações são particularmente importantes do ponto de vista algorítmico, pois elas nos fornecem uma maneira explícita de operar sobre o grupo. No entanto, a representação

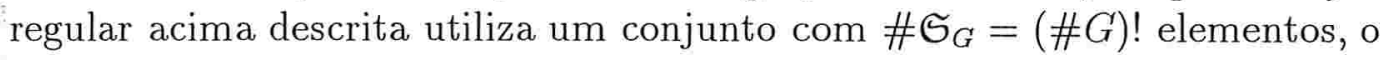
que é pouco razoável do ponto de vista computacional. Ainda assim, diversos grupos admitem representações como subgrupos de $\mathfrak{S}_{\Omega}$ para conjuntos $\Omega$ relativamente pequenos. Veremos que é possível resolver diversas questões interessantes de maneira eficiente, mesmo com grupos de ordem exponencial em $\# \Omega$.

\subsection{Grupos Transitivos}

Seja $G$ um grupo de permutações agindo sobre um conjunto $\Omega$. Para $x \in \Omega$ definimos a órbita de $x$ como sendo o conjunto $G(x)=\{\sigma(x) \mid \sigma \in G\}$. As órbitas particionam o conjunto $\Omega$; dizemos que $G$ é um grupo transitivo se $G$ possui apenas uma órbita.

Por exemplo, dado um grupo $G$ e um subgrupo $H \subset G$, a imagem da representação regular de $G$ associada a $H$ é transitiva. De fato, todas as classes laterais à esquerda de $H$ estão em uma mesma órbita, já que, dadas duas classes $g_{i} H$ e $g_{j} H$, temos que a imagem de $g_{j} g_{i}^{-1}$ é uma permutação que leva $g_{i} H$ em $g_{j} H$.

Dado $x \in \Omega$, definimos o estabilizador de $x$ como sendo o subgrupo de $G$ que fixa $x$, isto é, $\operatorname{Stab}(x)=\{\sigma \in G \mid \sigma(x)=x\}$. Temos a seguinte 
importante relação: para qualquer $x \in \Omega$,

$$
\# G=\# G(x) \cdot \# \operatorname{Stab}(x)
$$

A prova é simples: se $G(x)=\left\{\gamma_{1}(x), \ldots, \gamma_{r}(x)\right\}, \gamma_{i} \in G$, então $\gamma_{1}, \ldots, \gamma_{r}$ são representantes de classes laterais de $\operatorname{Stab}(x)$ em $G$, e o resultado segue.

Como encontrar a órbita $N$ de um elemento $x$ ? Observe que $N$ corresponde exatamente à componente conexa que contém $x$ no grafo cujos vértices são os elementos de $\Omega$ e em que $v w$ é uma aresta se, e somente se, existe um gerador $g$ de $G$ tal que $v=g(w)$. Assim, uma busca em largura é suficiente para obter $N$ :

1. No início, fazemos $N \leftarrow\{x\}$ e colocamos $x$ na fila $F$

2. retiramos o primeiro elemento $i$ de $F$ e, para cada gerador $g$ de $G$, calculamos $g(i)$. Se $g(i) \notin N$, nós o colocamos tanto em $N$ como no fim da fila.

3. se $F$ está vazia, o algoritmo termina. Senão repetimos o passo anterior.

$\mathrm{O}$ algoritmo acima acrescenta no máximo $n=\# \Omega$ elementos à fila, logo termina em $O(k n)$ passos, em que $k$ é o número de geradores de $G$. Repetindo o processo com outros elementos de $\Omega$, fora das órbitas até então consideradas, podemos obter a partição de $\Omega$ em órbitas de $G$. Naturalmente, o mesmo limitante de tempo continua valendo.

\subsection{Cadeias de Estabilizadores}

A fim de utilizar efetivamente a representação de um grupo como grupo de permutações, diversos algoritmos utilizam a chamada cadeia de estabilizadores : se $G$ é um grupo de permutações agindo sobre $\{1,2, \ldots, m\}$, seja $G_{0}=G$ e, para $1 \leq i \leq m-1, G_{i}$ é o subgrupo formado pelos elementos de $G$ que fixam cada um dos elementos $1,2, \ldots, i$. Temos

$$
G=G_{0} \supset G_{1} \supset G_{2} \supset \cdots \supset G_{m-1}=\{1\}
$$

Agora observe que representantes de classes laterais à esquerda do subgrupo $G_{i+1}$ de $G_{i}$ correspondem a permutações de $G_{i}$ que levam $i+1$ em cada elemento da órbita $G_{i}(i+1) \subset\{i+1, i+2, \ldots, m\}$. Assim, $\left[G_{i}: G_{i+1}\right] \leq m-i$. 
Naturalmente, a cadeia de estabilizadores de um grupo de permutações depende da particular escolha de numeração de seus elementos. Isto tem implicações importantes na eficiência dos algoritmos a seguir, já que suas performances variam para diferentes cadeias de estabilizadores.

Vejamos como utilizar cadeias de estabilizadores para resolver alguns problemas computacionais relacionados a grupos. Comecemos com a questão de decidir se uma dada permutação $\pi \in \mathfrak{S}_{m}$ está ou não em $G$.

Inicialmente, suponha que temos à nossa disposição conjuntos $C_{i}$ 's (eventualmente incompletos) de representantes de classes laterais à esquerda de $G_{i}$ módulo $G_{i+1}$. Definimos o seguinte procedimento, que chamaremos de "filtragem", e que tenta escrever $\pi$ como produto de elementos de $C_{i}$ 's: dada uma permutação $\pi \in \mathfrak{S}_{m}$, a filtragem de $\pi$ devolve uma permutação $\pi^{\prime}$, um índice $i$ e uma seqüência $\gamma_{0}, \gamma_{1}, \ldots, \gamma_{i-1}$ tal que

- $\gamma_{k} \in C_{k}$ para $0 \leq k<i$

- $\pi=\gamma_{0} \gamma_{1} \ldots \gamma_{i-1} \pi^{\prime}$

- $\pi^{\prime}=1$ ou então $\pi^{\prime}$ não é congruente a nenhum elemento de $C_{i}$ módulo $G_{i+1}$.

Formalmente, temos a seguinte descrição de uma filtragem:

$$
\begin{aligned}
& \text { procedimento filtragem }(\pi) \\
& s \leftarrow \emptyset \\
& i \leftarrow 0 \text {; continuar } \leftarrow \text { verdadeiro } \\
& \text { enquanto } i \leq m-2 \text { e continuar, } \\
& \text { faça } \\
& \text { continuar } \leftarrow \text { falso } \\
& \text { para cada } \gamma \in C_{i}, \\
& \text { se } \gamma^{-1} \pi(i+1)=i+1, \\
& \text { então } \\
& s \leftarrow(s, \gamma) ; \pi \leftarrow \gamma^{-1} \pi \\
& i \leftarrow i+1 ; \text { continuar } \leftarrow \text { verdadeiro } \\
& \text { devolva }(\pi, i, s) .
\end{aligned}
$$

A observação crucial aqui é que se $\pi$ está em $G=G_{0}$, então ele está em alguma das classes laterais à esquerda de $G_{1}$, digamos em $\gamma_{0} G_{1}$. Logo $\gamma_{0}^{-1} \pi \in G_{1}$ e podemos repetir o procedimento com $G_{1}$ no lugar de $G_{0}$ e assim sucessivamente. Eventualmente, teremos $\gamma_{i-1}^{-1} \ldots \gamma_{0}^{-1} \pi \notin \gamma_{i} G_{i+1}$ para 
todo $\gamma_{i} \in C_{i}$, mostrando que $\pi$ não está em $G$, ou $\gamma_{m-2}^{-1} \gamma_{m-3}^{-1} \ldots \gamma_{0}^{-1} \pi \in$ $G_{m-1}=\{1\}$, ou seja, que $\pi=\gamma_{0} \ldots \gamma_{m-3} \gamma_{m-2} \in G$. Em particular, temos que $\bigcup_{0 \leq i \leq m-2} C_{i}$ é um conjunto de geradores de $G$ e como $\# C_{i} \leq m-i$, concluímos que um grupo de permutações de grau $m$ admite um conjunto de geradores com no máximo $m(m+1) / 2$ elementos.

Naturalmente, nem sempre podemos contar com a boa sorte de possuir um conjunto de representantes de classes laterais. O que fazer então? A idéia é utilizar a filtragem para construir um tal conjunto de representantes: ao filtrarmos um elemento de $\pi \in G$, devemos obter sempre $\pi^{\prime}=1$. Assim, se $\pi^{\prime} \neq 1$, temos um representante de classe ausente, que acrescentamos a $C_{i}$. É neste princípio que se baseia o algoritmo de Sims:

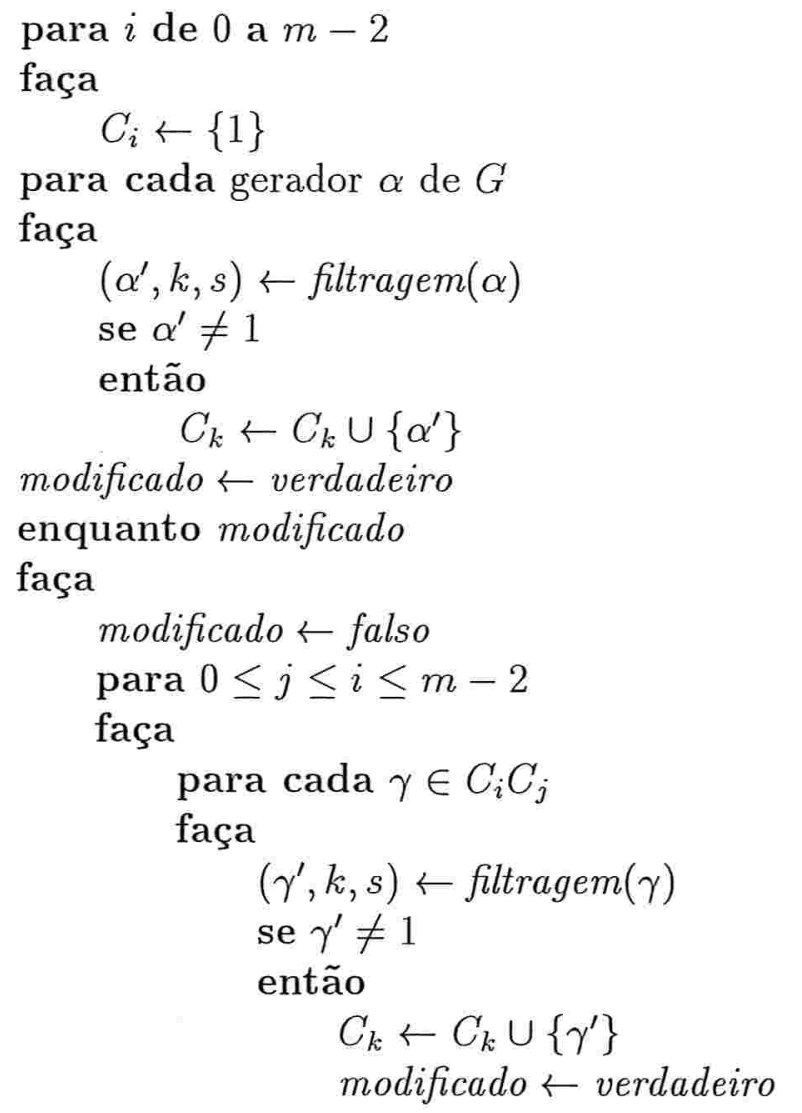

Observe que sabemos de antemão que $\# C_{i} \leq m-i$, logo o procedimento não pode modificar os $C_{i}$ 's indefinidamente e o algoritmo termina. Para verificar que os $C_{i}$ 's são realmente conjuntos de representantes de classes, é 
suficiente verificar que $G=C_{0} C_{1} \ldots C_{m-2}$, uma vez que a filtragem mantém os elementos de $C_{i}$ incongruentes entre si módulo $G_{i+1}$.

Temos $C_{i} \subset G_{i}$ e $C_{j} \subset G_{j}$, logo se $i \geq j$, temos $C_{i} C_{j} \subset G_{j}$. Assim, as filtragens de $C_{i} C_{j}$ acima garantem que

$$
C_{i} C_{j} \subset C_{j} C_{j+1} \ldots C_{m-2}
$$

para $i \geq j$.

Assim, para concluirmos que $G=C_{0} C_{1} \ldots C_{m-2}$, basta notar que os geradores de $G$ certamente pertencem a $C_{0} C_{1} \ldots C_{m-2}$ e a expressão acima garante que $C_{0} C_{1} \ldots C_{m-2}$ é fechado por produto. De fato,

$$
\begin{aligned}
C_{0} \ldots C_{m-2} C_{0} \ldots C_{m-2} & \subset C_{0} \ldots C_{m-3} C_{0} \ldots C_{m-2} C_{1} \ldots C_{m-2} \\
& \subset C_{0} \ldots C_{m-4} C_{0} \ldots C_{m-2} C_{1} \ldots C_{m-2} C_{1} \ldots C_{m-2} \\
& \vdots \\
& \subset C_{0} \times \text { produto de } C_{i}{ }^{\prime} \text { s, } i \geq 1
\end{aligned}
$$

Podemos supor, por hipótese de indução, que o produto de $C_{i}$ 's com $i \geq 1$ está contido em $C_{1} C_{2} \ldots C_{m-2}$, o que encerra a demonstração.

$\mathrm{O}$ algoritmo de Sims, ao construir conjuntos de representantes de classes laterais para os subgrupos da cadeia de estabilizadores, permite não só responder a questão da pertinência de um elemento a $G$, mas também determinar a ordem de um grupo de permutações $G$ dado por geradores: observe que a representação de um elemento de $G$ como $\gamma_{0} \ldots \gamma_{m-1} \gamma_{m-2}, \gamma_{i} \in C_{i}$, é única e, portanto,

$$
\# G=\# C_{0} \cdot \# C_{1} \cdots \# C_{m-2}
$$

Qual a complexidade deste algoritmo? Inicialmente, note que uma filtragem executa o teste $\gamma^{-1} \pi(i+1)=i+1$ no máximo $\# C_{i} \leq m-i$ vezes, logo uma filtragem gasta um tempo $m+(m-1)+\cdots+2=O\left(m^{2}\right)$.

Seja $k$ o número de geradores de $G$. A filtragem dos geradores gasta tempo $O\left(\mathrm{~km}^{2}\right)$. Novamente como $\# C_{i} \leq m-i$, sabemos ainda que os conjuntos $C_{i}$ 's são modificados no máximo $O\left(\mathrm{~m}^{2}\right)$ vezes. Assim, o número de filtragens de elementos de $C_{i} C_{j}, i \geq j$, é limitado por

$$
O\left(m^{2}\right) \sum_{0 \leq j \leq i \leq m-2}(m-i)(m-j)=O\left(m^{6}\right)
$$

Portanto a complexidade final é dada por $O\left(k m^{2}+m^{8}\right)$. 
$\mathrm{Na}$ verdade, o algoritmo, na forma apresentada acima, realiza várias filtragens redundantes, uma vez que cada elemento $\gamma$ de $C_{i} C_{j}, i \geq j$, só precisa ser considerado uma única vez: uma segunda filtragem de $\gamma$ certamente resultará em $\gamma^{\prime}=1$. Uma reordenação cuidadosa das filtragens pode melhorar o limitante de tempo para $O\left(\mathrm{~km}^{2}+\mathrm{m}^{5} \lg \lg \mathrm{m}\right)$. Para maiores detalhes, consulte [Hof]. Referimos-nos também a esta obra para diversos outros algoritmos, baseados em cadeias de estabilizadores.

Por fim, como mais uma aplicação de cadeia de estabilizadores, descrevemos a redução polinomial que ficamos devendo no capítulo anterior: dado um grafo $X$, vamos obter geradores para $G=\operatorname{Aut}(X)$ dispondo de um algoritmo que testa isomorfismo entre grafos rotulados.

Numere os vértices de $X$ de 1 a $m=\# V(X)$ e considere a cadeia de estabilizadores associada:

$$
G=G_{0} \supset G_{1} \supset G_{2} \supset \cdots \supset G_{m-1}=\{1\}
$$

Como visto no capítulo anterior, o teste de isomorfismo de grafos rotulados pode ser utilizado para obter a órbita $G_{r}(r+1)$. Além disso, para. cada elemento $x$ desta órbita, este mesmo teste permite obter explicitamente uma permutação que leva $r+1$ em $x$. Note que estas permutações são representantes de classes laterais de $G_{r}$ módulo $G_{r+1}$. A união destes representantes de classes para $r=0,1, \ldots, m-2$ fornece-nos o conjunto de geradores procurado.

\subsection{Subgrupos de Sylow}

Aqui, temos outra aplicação das idéias expostas acima. Dados geradores para um grupo de permutações $G \subset \mathfrak{S}_{n}$, veremos como determinar geradores para um $p$-subgrupo de Sylow de $G$. Sendo $\# G=p^{s} m$, em que $m$ não é múltiplo de $p$, o algoritmo será polinomial desde que $m$ seja polinomial em $n$. Utilizaremos este algoritmo no próximo capítulo, em que tal restrição se aplica.

Dados geradores para um $p$-subgrupo $P$ e um elemento $\sigma \in G$, é fácil testar se eles estão em algum subgrupo de Sylow comum: basta calcular a ordem de $\langle P, \sigma\rangle$ e verificar se ela é uma potência de $p$ ou não. Utilizando esta observação preliminar, vejamos como resolver o problema. Construiremos simultaneamente um conjunto $\Pi$ de geradores para um $p$-subgrupo de Sylow 
$P$ e um conjunto $C$ de representantes de classe laterais à esquerda de $G$ módulo $P$.

Inicialmente, definimos uma $p$-filtragem de um elemento $\alpha \in G$, que consiste no seguinte procedimento:

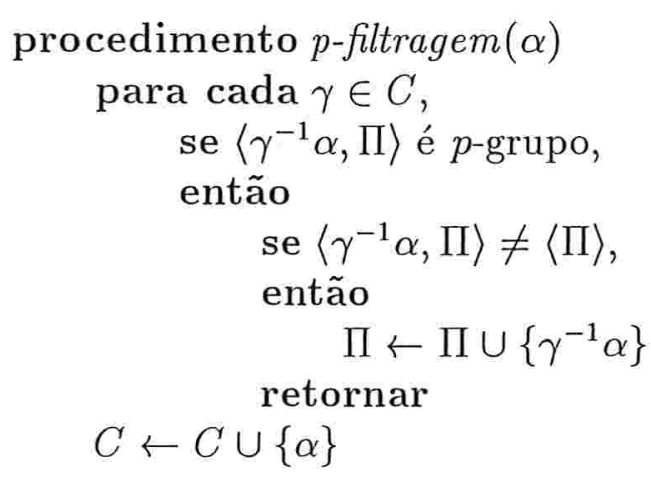

Observe que a condição $\left\langle\gamma^{-1} \alpha, \Pi\right\rangle \neq\langle\Pi\rangle$ se resume simplesmente a $\gamma^{-1} \alpha \notin$ $\langle\Pi\rangle$.

Em qualquer instante, temos que $\langle\Pi\rangle$ é um $p$-subgrupo e $C$ é um conjunto de elementos dois a dois incongruentes modulo qualquer subgrupo de Sylow que contenha $\langle\Pi\rangle$. Se $\alpha$ não é congruente a nenhum $\gamma \in C$ módulo um subgrupo de Sylow que contém $\langle\Pi\rangle$, acrescentamos $\alpha$ aos representantes de classe laterais do subgrupo de Sylow que está sendo construído. Caso contrário, se $\alpha$ é congruente a $\gamma$ módulo algum subgrupo de Sylow que contenha $\langle\Pi\rangle$, isto é, se $\left\langle\gamma^{-1} \alpha, \Pi\right\rangle$ é um $p$-grupo, ao fazermos $\Pi \leftarrow \Pi \cup\left\{\gamma^{-1} \alpha\right\}$, passamos a considerar apenas aqueles subgrupos de Sylow que contêm $\left\langle\Pi, \gamma^{-1} \alpha\right\rangle$.

Agora, vejamos como uma pequena modificação do algoritmo de Sims nos permite construir um subgrupo de Sylow. Seja $\Phi$ um conjunto de geradores de $G$. A idéia é aplicar uma $p$-filtragem nos elementos de $\Phi C$. Obtemos o seguinte algoritmo:

$$
\begin{aligned}
& \Pi \leftarrow \emptyset ; C \leftarrow\{1\} \\
& \text { faça } \quad \text { para cada } \alpha \in \Phi C, \\
& \quad \quad \text { - filtragem }(\alpha) \\
& \text { enquanto houver modificações em } C
\end{aligned}
$$

Note que como os elementos de $C$ são dois a dois incongruentes módulo um certo subgrupo de Sylow, $\# C \leq m$ e, desta forma, as $p$-filtragens acima só podem alterar $C$ no máximo $m$ vezes. Assim, o algoritmo termina, e em tempo polinomial se $m=O\left(n^{c}\right)$ para alguma constante $c$. 
Como no algoritmo de Sims, as $p$-filtragens de elementos de $\Phi C$ garantem que $\Phi C \subset C\langle\Pi\rangle$ e, desta forma, $\Phi^{k} C \subset C\langle\Pi\rangle$, logo $G=C\langle\Pi\rangle$ e $C$ é um conjunto de representantes de classe à esquerda de $\langle\Pi\rangle$. Como $\langle\gamma, \Pi\rangle$ não é um $p$-grupo para nenhum $\gamma \in C, \gamma \neq 1$, concluímos que $\langle\Pi\rangle$ é um subgrupo de Sylow.

\subsection{Grupos Primitivos}

Às vezes, é possível obter uma representação (não necessariamente fiel) de um grupo de permutações como grupo de permutações de um conjunto menor. Isto ocorre, por exemplo, quando o grupo é intransitivo: podemos estudar a ação deste grupo sobre uma de suas órbitas. Mesmo quando o grupo é transitivo, tal representação pode existir. É aqui que entra em cena o conceito de primitividade .

Seja $G$ um grupo de permutações transitivo agindo sobre um conjunto $\Omega$. Uma partição $\mathcal{P}$ de $\Omega$ é dita não trivial se, para todo $B \in \mathcal{P}, B \neq \Omega$ e $\# B>1$. Dizemos que $G$ é um grupo imprimitivo se existir uma partição não trivial $\mathcal{P}$ tal que, para quaisquer $\sigma, \tau \in G$, e para qualquer bloco $B \in \mathcal{P}$, $\sigma(B)=\tau(B)$ ou $\sigma(B) \cap \tau(B)=\emptyset$. Neste caso, dizemos que a partição $\mathcal{P}$ é um sistema de imprimitividade para $G$. Observe que grupos imprimitivos agem permutando os blocos de um sistema de imprimitividade. Se $G$ não for imprimitivo, diremos que $G$ é primitivo (grande surpresa!)

Por exemplo, seja $K$ um corpo com mais de 2 elementos e seja $\Omega=$ $K^{n}-\{0\}$. O grupo $G L(K, n)$ das transformações lineares inversíveis de $K^{n}$ age transitivamente sobre $\Omega$, porém não é primitivo, uma vez que os subespaços lineares menos a origem formam um sistema de imprimitividade para $G L(K, n)$.

Note que, para verificar que uma partição não trivial $\mathcal{P}$ de $\Omega$ é um sistema de imprimitividade para $G$, basta nos certificarmos de que, para qualquer $\sigma \in G$ e qualquer bloco $B \in \mathcal{P}, \sigma(B)=B$ ou $\sigma(B) \cap B=\emptyset$. De fato, se, para $\sigma, \tau \in G, \sigma(B) \cap \tau(B) \neq \emptyset$, então $\tau^{-1} \sigma(B) \cap B \neq \emptyset$, logo $\tau^{-1} \sigma(B)=B$, isto é, $\sigma(B)=\tau(B)$.

O seguinte critério é muitas vezes útil na identificação de grupos primitivos.

Teorema 2.2 Seja $G \subset \mathfrak{S}_{\Omega}$ um grupo transitivo e sejam $x \in \Omega$ e $H=$ $\operatorname{Stab}(x)$. Então $G$ é primitivo se, e somente se, $H$ for um subgrupo maximal 
de $G$. Além disso, se $K$ é um subgrupo estritamente entre $G$ e $H$, então as órbitas de $K$ formam um sistema de imprimitividade para $G$.

Demonstração Inicialmente, observe que, como $G$ é transitivo, podemos identificar cada $y \in \Omega$ com a classe lateral $\sigma_{y} H$, em que $\sigma_{y} \in G$ é tal que $\sigma_{y}(x)=y$. Note que $\sigma_{y} H$ é independente da escolha do elemento $\sigma_{y}$. Nesta identificação, a ação de $G$ corresponde justamente à da representação regular de $G$ associada a $H$.

Suponha que $H \subsetneq K \subsetneq G$. As órbitas de $K$ correspondem às classes laterais de $K$ na representação regular, logo formam um sistema de imprimitividade para $G$. Logo, se $G$ é primitivo, então $H$ é maximal. Reciprocamente, se $H$ é maximal e $B_{i}, 1 \leq i \leq r$, é um sistema de imprimitividade para $G$, seja $K$ o subgrupo que estabiliza o bloco $B_{1}$ que contém $x$. Note que se $\tau \in H, x \in \tau\left(B_{1}\right) \cap B_{1}$, logo devemos ter $\tau\left(B_{1}\right)=B_{1}$, ou seja, $H \subset K$. Como $[G: K]=r$ e $[G: H]=\# \Omega>r$, temos $H \subsetneq K \subsetneq G$, contrariando a maximalidade de $H$. Logo $G$ é primitivo, o que encerra a demonstração.

Vamos agora descrever um algoritmo que, em tempo polinomial, decide se um grupo transitivo $G$ é ou não primitivo e, em caso negativo, constrói um sistema de imprimitividade minimal, isto é, um sistema sobre o qual $G$ age primitivamente (não podemos juntar blocos para formar outro sistema de imprimitividade). O número de blocos em um sistema de imprimitividade minimal não é, em geral, unicamente determinado.

Sejam $a, b \in \Omega$ e considere o grafo $X$ cujos vértices são os elementos de $\Omega$ e em que $v w$ é uma aresta se, e somente se, existe $\sigma \in G$ tal que $\sigma(a)=v$ e $\sigma(b)=w$. Sejam $X_{i}, 1 \leq i \leq t$, as componentes conexas de $X$, sendo $X_{1}$ aquela que contém $a, b$.

Se $G$ é imprimitivo e $a, b$ pertencem a um mesmo bloco $B$ de um sistema de imprimitividade, então $V\left(X_{1}\right) \subset B$. De fato, se $v_{1} v_{2} \ldots v_{l-1} v_{l}$ é um caminho de $X_{1} \operatorname{com} v_{1} \in\{a, b\}$ e, digamos, $v_{l-1}=\sigma(a)$ e $v_{l}=\sigma(b)$, podemos supor, por hipótese de indução, que $v_{l-1} \in B$. Mas então $v_{l-1} \in \sigma(B) \cap B$, portanto $v_{l} \in \sigma(B)=B$.

Por outro lado, temos que se, para algum par $a, b$, o grafo $X$ for desconexo, então os blocos $V\left(X_{i}\right)$ formam um sistema de imprimitividade para $G$. Note que se $v w \in E(X), \operatorname{com} v=\sigma(a)$ e $w=\sigma(b)$, então $\tau(v) \tau(w)=\tau \sigma(a) \tau \sigma(b) \in$ $E(X)$ para todo $\tau \in G$. Desta forma, $G$ age permutando as componentes conexas de $X$ e o resultado segue. 
Temos, portanto, que $G$ será imprimitivo se, e somente se, $X$ for um grafo desconexo para alguma escolha de $a, b$. Isto pode ser testado alterando-se convenientemente o algoritmo da seção 2.2, em tempo $O\left(k n^{2}\right)$, em que $n=\# \Omega$ e $k$ é o número de geradores de $G$. Repetindo este teste no máximo $\left(\begin{array}{l}n \\ 2\end{array}\right)=O\left(n^{2}\right)$ vezes, decidimos se o grupo é ou não primitivo em tempo $O\left(k n^{4}\right)$. Caso seja imprimitivo, podemos repetir o procedimento recursivamente, substituindo $\Omega$ pelo sistema de imprimitividade, até obtermos finalmente o sistema minimal procurado. O tempo gasto $T(n)$ satisfaz a recursão $T(n)=T(n / 2)+k n^{4}$, cuja solução nos fornece o limitante polinomial $T(n)=O\left(k n^{4} \lg n\right)$.

\subsection{Representações Infiéis}

Até o presente momento, os algoritmos considerados trabalharam apenas com a representação fiel naturalmente associada a um grupo de permutações. Entretanto, como mostramos na seção anterior, é importante também considerar representações infiéis destes grupos. Aqui, esboçaremos como modificar os algoritmos vistos para trabalharem com representações que não são necessariamente fiéis.

Seja $G$ um grupo e $\pi: G \rightarrow \mathfrak{S}_{m}$ uma representação de $G$. Seja ainda $K=\operatorname{ker} \pi$. Podemos construir uma cadeia de estabilizadores "módulo $K$ " da seguinte forma: seja $G^{0}=G$ e $G^{i}$ o subgrupo de $G$ que estabiliza cada elemento $1,2, \ldots, i$. Temos

$$
G=G^{0} \supset G^{1} \supset G^{2} \supset \cdots \supset G^{m-1}=K
$$

$\operatorname{com}\left[G^{i}: G^{i+1}\right] \leq m-i$.

Agora, se para todo $g \in G$ e $i \in\{1,2, \ldots, m\}$ é possível testar a condição $\pi(g)(i)=i$ em tempo polinomial, então a cadeia de estabilizadores acima pode ser utilizada para responder questões relativas à ação de $G$ sobre $\{1,2, \ldots, m\}$. Para isto, basta alterar os algoritmos anteriores de modo a incluir este novo teste. Como exemplo desta técnica e também para uso futuro, vamos mostrar como determinar, em tempo polinomial, geradores para o subgrupo de um grupo imprimitivo que estabiliza todos os blocos de um sistema de imprimitividade.

Seja $G \subset \mathfrak{S}_{n}$ um grupo imprimitivo e seja $B_{i}, 1 \leq i \leq m$, um sistema de imprimitividade para $G$. Considerando a ação de $G$ sobre os blocos do sistema de imprimitividade, montamos uma cadeia de estabilizadores como 
acima, em que $G^{i}$ denota o subgrupo dos elementos que estabilizam cada um dos primeiros $i$ blocos.

Uma vez conhecidos geradores para $G^{i}$, vamos mostrar como obter geradores para o subgrupo $G^{i+1}$ de $G^{i}$. Para isto, modificamos a cadeia de estabilizadores no algoritmo de Sims. Seja $H_{j}$ o subgrupo de $G^{i+1}$ que fixa os primeiros $j$ inteiros, agora segundo a ação de sobre $\mathfrak{S}_{n}$ (e não a sobre os blocos de imprimitividade). A nova cadeia é dada por

$$
G^{i} \supset G^{i+1}=H_{0} \supset H_{1} \supset H_{2} \supset \cdots \supset H_{m-1}=\{1\}
$$

A filtragem deve ser alterada de modo a incluir o termo $G^{i}$ módulo $G^{i+1}$ :

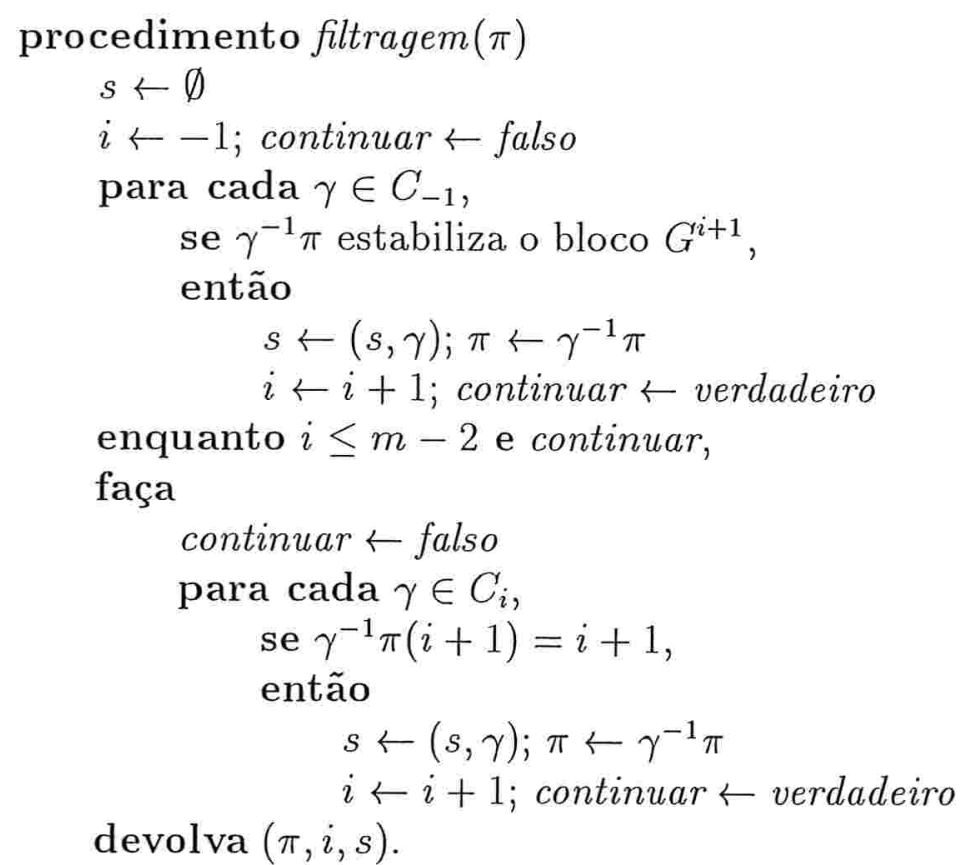

Ao final da execução do algoritmo modificado, a união dos representantes de classes laterais de $H_{j}$ módulo $H_{j+1}$ fornecerão os geradores de $G^{i+1}$ procurados. Note que, como $\left[G^{i}: G^{i+1}\right]$ é polinomial em $m$, a análise do algoritmo de Sims continua válida e o algoritmo resultante termina em tempo polinomial. Repetindo este procedimento para $i=0,1, \ldots$, obteremos no final geradores para o subgrupo que estabiliza todos os blocos em tempo polinomial, como afirmamos. 


\section{Capítulo 3}

\section{O algoritmo de Luks}

Neste capítulo, apresentamos o algoritmo de Luks [Luk], que testa se dois grafos são isomorfos em tempo polinomial no número de vértices, desde que os graus destes sejam limitados por uma constante. De fato, esta última condição impõe restrições nos possíveis grupos que podem ser grupos de automorfismos de grafos e é a análise cuidadosa da estrutura destes grupos a responsável pela limitação polinomial.

Iniciamos a apresentação com uma redução do problema original a um problema computacional de grupos. Em seguida, veremos como os conceitos de primitividade e transitividade de um grupo de permutações podem ser explorados para obter um procedimento recursivo para resolver este problema reduzido. Por fim, mostraremos que este algoritmo tem a complexidade desejada, uma vez que demonstrarmos a existência de um certo subgrupo de Sylow de índice pequeno. Esta é a parte mais difícil e a que consumirá maiores esforços, e a que depende mais profundamente da teoria de grupos de permutações.

\subsection{Reduzindo o problema}

Nosso objetivo aqui será provar que o problema de testar se dois grafos são ou não isomorfos pode ser reduzido, em tempo polinomial, ao problema de, dado um grupo de permutações agindo sobre um conjunto colorido, obter geradores para o subgrupo das permutações que preservam cores.

Inicialmente, observemos que testar isomorfismo de grafos pode ser polinomialmente reduzido ao seguinte problema: dado um grafo $X$ e uma de 
suas arestas $e$, determinar geradores para $\operatorname{Aut}_{e}(X)$, o subgrupo de $\operatorname{Aut}(X)$ que fixa $e$ (eventualmente trocando seus extremos). Vejamos como.

Ao testar se dois grafos $X_{1}$ e $X_{2}$ são ou não isomorfos, podemos, substituindo-os pelos complementares se necessário, supor que ambos são conexos. Agora, para cada par de arestas $e_{1}$ de $X_{1}$ e $e_{2}$ de $X_{2}$, basta descobrir se há um isomorfismo entre $X_{1}$ e $X_{2}$ que leva $e_{1}$ em $e_{2}$, já que o número de tais pares é polinomial no número de vértices. Isto pode ser feito da seguinte forma: crie dois novos vértices $v_{1}$ em $e_{1}$ e $v_{2}$ em $e_{2}$, ligue-os por uma nova aresta $e$ e considere o grafo $X$ resultante (note que esta construção mantém o grau máximo dos vértices de $X_{1}$ e $X_{2}$ ). Haverá um isomorfismo entre $X_{1}$ e $X_{2}$ que leva $e_{1}$ em $e_{2}$ se, e somente se, existir um automorfismo que fixa $e$, trocando seus extremos. Esta condição pode ser facilmente testada se temos um conjunto de geradores para $\operatorname{Aut}_{e}(X)$ : basta verificar se há algum gerador que troca os extremos $e$.

Como calcular $\operatorname{Aut}_{e}(X)$ ? A idéia é utilizar uma série de aproximações $\operatorname{Aut}_{e}\left(X_{r}\right)$, em que $X_{r}$ é o subgrafo de $X$ formado por todos os vértices e arestas que aparecem em caminhos contendo a aresta $e$ e cujo comprimento é menor ou igual a $r$ para $r=1,2, \ldots$ Assim, por exemplo, $X_{1}$ é o subgrafo formado pela aresta $e$ e seus vértices, enquanto que $X_{n-1}=X(n=\# V(X))$.

Podemos definir um homomorfismo

$$
\pi_{r}: \operatorname{Aut}_{e}\left(X_{r+1}\right) \rightarrow \operatorname{Aut}_{e}\left(X_{r}\right)
$$

restringindo um automorfismo de $\operatorname{Aut}_{e}\left(X_{r+1}\right)$ a $X_{r}$. Note que se pudermos obter geradores para o kernel e para a imagem de $\pi_{r}$, então podemos obter geradores para $\operatorname{Aut}_{e}\left(X_{r+1}\right)$ da seguinte forma: sejam $s_{i} \in \operatorname{Aut}_{e}\left(X_{r+1}\right)$ geradores para o kernel e $t_{j} \in \operatorname{Aut}_{e}\left(X_{r+1}\right)$ elementos tais que $\pi_{r}\left(t_{j}\right)$ gerem a imagem. Então $s_{i}$ e $t_{j}$ geram $\operatorname{Aut}_{e}\left(X_{r+1}\right)$. De fato, dado um automorfismo

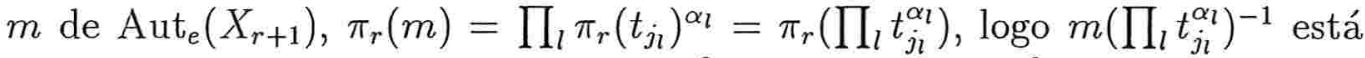
no kernel, ou seja, $m\left(\prod_{l} t_{j_{l}}^{\alpha_{l}}\right)^{-1}=\prod_{k} s_{i_{k}}^{\beta_{k}} \Longleftrightarrow m=\prod_{k} s_{i_{k}}^{\beta_{k}} \prod_{l} t_{j_{l}}^{\alpha_{l}}$.

Doravante, consideraremos apenas grafos com vértices de grau limitado por uma constante $t$. Seja $A$ a coleção de todos os subconjuntos não vazios de $V\left(X_{r}\right)$ de cardinalidade menor ou igual a $t-1$. Cada vértice em $V\left(X_{r+1}\right)-$ $V\left(X_{r}\right)$ está ligado a um ou mais vértices de $V\left(X_{r}\right)$, o que pode ser descrito pela seguinte função "paternidade" (ou maternidade, se preferir):

$$
\begin{gathered}
f: V\left(X_{r+1}\right)-V\left(X_{r}\right) \rightarrow A \\
f(v)=\left\{w \in V\left(X_{r}\right) \mid v w \in E(X)\right\}
\end{gathered}
$$


Por definição, um automorfismo $s \in \operatorname{Aut}_{e}\left(X_{r+1}\right)$ está no kernel de $\pi_{r}$ se, e somente se, fixa todos os vértices de $X_{r}$. Assim, $s$ permuta somente os vértices "irmãos" entre si em $V\left(X_{r+1}\right)-V\left(X_{r}\right)$, já que se $v \in V\left(X_{r+1}\right)-$ $V\left(X_{r}\right)$, então para todo vértice $w$, pai de $v, w \in f(v) \subset V\left(X_{r}\right), s(v) s(w)=$ $s(v) w$ é uma aresta de $X_{r+1}$. Reciprocamente, qualquer permutação deste tipo fornece-nos um automorfismo em ker $\pi_{r}$. Portanto este grupo é o produto direto de grupos simétricos de irmãos:

$$
\operatorname{ker} \pi_{r}=\underset{a \in A}{\chi} \mathfrak{S}_{f^{-1}(a)}
$$

E quanto à imagem? Bem, esta é a parte difícil do problema. Vamos mostrar que podemos resolvê-lo uma vez que tivermos à nossa disposição um algoritmo que, dado um conjunto colorido $S$ e um grupo de permutações de $S$, determina o subgrupo das permutações que preservam cores.

Seja $A_{i}$ a coleção de conjuntos de pais com $i$ filhos, isto é, $A_{i}=\{a \in$ $\left.A \mid \# f^{-1}(a)=i\right\}$ para $0 \leq i \leq t-1$ (lembrando que nosso grafo tem grau limitado por $t$ ). Seja ainda $A^{\prime}$ a coleção de "novas" arestas, arestas em $E\left(X_{r+1}\right)-E\left(X_{r}\right)$ incidindo somente sobre vértices de $X_{r}$. Uma permutação $s \in \operatorname{Aut}_{e}\left(X_{r}\right)$ está na imagem de $\pi_{r}$ se puder ser estendida a uma permutação

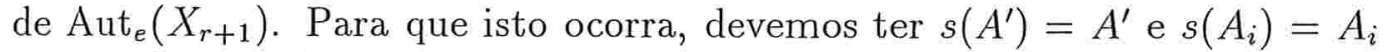
para todo $0 \leq i \leq t-1$. E uma permutação satisfazendo estas condições pode realmente ser estendida a um automorfismo de $\operatorname{Aut}_{e}\left(X_{r+1}\right)$ : se $a \in A_{i}$ tem $i$ filhos, então $s(a) \in A_{i}$ também, logo mapeamos os filhos de $a$ nos filhos de $s(a)$ de qualquer jeito. Isto nos fornece a extensão procurada.

Permutando os vértices de $X_{r}$, os elementos de $A$ também são permutados e a imagem de $\pi_{r}$ corresponde justamente às permutações que estabilizam os subconjuntos $A_{i}$ e $A^{\prime}$. Como os $A_{i}$ 's são dois a dois disjuntos, basta olhar para as intersecções dos $A_{i}{ }^{\prime}$ s com $A^{\prime}$ e seu complementar e colorir $A$ de $2 t$ cores:

$$
\begin{aligned}
& A_{0} \cap A^{\prime}, A_{1} \cap A^{\prime}, \ldots, A_{t-1} \cap A^{\prime}, \\
& A_{0}-A^{\prime}, A_{1}-A^{\prime}, \ldots, A_{t-1}-A^{\prime}
\end{aligned}
$$

A imagem é o subgrupo que preserva estas cores.

Sendo $n=\# V(X)$, note que $A$ possui

$$
\left(\begin{array}{l}
n \\
1
\end{array}\right)+\left(\begin{array}{l}
n \\
2
\end{array}\right)+\cdots+\left(\begin{array}{c}
n \\
t-1
\end{array}\right)=O\left(n^{t-1}\right)
$$


elementos, que é polinomial em $n$ (já que $t$ é fixado). Como o número de homomorfismos $\pi_{r}$ considerados é menor ou igual a $n$, obtivemos assim nossa redução polinomial do problema de determinar $\operatorname{Aut}_{e}(X)$ ao de calcular automorfismos de um conjunto colorido.

\subsection{A classe $\Gamma_{k}$}

Recordemos que uma série de composição para um grupo $G$ é uma cadeia de subgrupos da forma

$$
\{1\}=G_{m} \triangleleft G_{m-1} \triangleleft \cdots \triangleleft G_{1} \triangleleft G_{0}=G
$$

em que os fatores $G_{i} / G_{i+1}$ são simples. Pelo teorema de Jordan-Hölder, estes fatores independem da escolha da série de composição e são, portanto, invariantes do grupo $G$.

A natureza dos kernels dos homomorfismos $\pi_{r}$ sugere estudar grupos tais que todos os seus fatores de composição são subgrupos de $\mathfrak{S}_{k}$ para algum $k$ fixo. Seguindo Babai, chamaremos a classe destes grupos de $\Gamma_{k}$. Observe que

$$
\# G=\#\left(G_{0} / G_{1}\right) \cdot \#\left(G_{1} / G_{2}\right) \cdots \#\left(G_{m-1} / G_{m}\right)
$$

e, portanto, os fatores primos de \#G não excedem $k$, o que, como veremos, será crucial na demonstração de que o algoritmo de Luks gasta tempo polinomial.

A introdução da classe $\Gamma_{k}$ foi motivada pelo estudo do kernel de $\pi_{r}$, e nosso principal objetivo aqui é demonstrar o

Teorema 3.1 Para todo $r, \operatorname{Aut}_{e}\left(X_{r}\right) \in \Gamma_{t-1}$. Em particular, $\operatorname{Aut}_{e}(X) \in$ $\Gamma_{t-1}$.

Vamos estudar a classe $\Gamma_{k}$ mais detalhadamente. Em primeiro lugar, observemos que se $N$ é um subgrupo normal de $G$ e $N$ e $G / N$ estão em $\Gamma_{k}$, então $G \in \Gamma_{k}$ também, já que dadas séries de composição para $N$ e $G / N$

$$
\begin{gathered}
\{1\}=N_{r} \triangleleft \cdots \triangleleft N_{1} \triangleleft N_{0}=N \\
\{1\}=G_{s} / N \triangleleft \cdots \triangleleft G_{1} / N \triangleleft G_{0} / N=G / N,
\end{gathered}
$$

$\operatorname{com} G_{i} \supset N$, temos que

$$
\{1\}=N_{r} \triangleleft \cdots \triangleleft N_{0}=N=G_{s} \triangleleft \cdots \triangleleft G_{0}=G
$$


é uma série de composição para $G$. Em particular, se $G_{1}, G_{2} \in \Gamma_{k}$, então $G_{1} \times G_{2} \in \Gamma_{k}$, pois $\left(G_{1} \times G_{2}\right) / G_{1} \cong G_{2}$.

Em seguida, vamos mostrar que subgrupos de grupos em $\Gamma_{k}$ também estão em $\Gamma_{k}$. Antes de mais nada, verifiquemos que qualquer subgrupo $G$ de $\mathfrak{S}_{k}$ está em $\Gamma_{k}$. Basta mostrarmos que se $N$ é um subgrupo normal próprio de $G$ com $G / N$ simples, então $G / N$ é um subgrupo de $\mathfrak{S}_{k}$. Para isto, seja $\left\{G_{i}\right\}$ a cadeia de estabilizadores de $G$, em que $G_{i}$ é o subgrupo de $G$ que fixa cada elemento de $\{1,2, \ldots, i\}$. Considere agora a seguinte cadeia:

$$
N=G_{k-1} N \subset \cdots \subset G_{1} N \subset G_{0} N=G
$$

Seja $j$ o menor índice tal que $G_{j+1} N \subsetneq G_{j} N$. Observe que $G_{j} N=G$ e que

$$
1<\left[G_{j} N: G_{j+1} N\right] \leq\left[G_{j}: G_{j+1}\right] \leq k-j \leq k
$$

Para encerrarmos a demonstração, vamos mostrar que a representação regular $\phi$ de $G / N=G_{j} N / N$ associada a $G_{j+1} N / N$ é fiel. Do fato de que $G / N$ é simples, temos $\operatorname{ker} \phi=\{1\}$ ou $\operatorname{ker} \phi=G / N$. Por outro lado, a imagem de $\phi$ age transitivamente sobre as classes laterais, portanto esta imagem é não trivial. Assim, $\operatorname{ker} \phi=\{1\}$, como queríamos demonstrar.

Voltemos ao caso geral. Seja $G \in \Gamma_{k}$ e $H$ um subgrupo de $G$. Considere uma série de composição para $G$ :

$$
\{1\}=G_{m} \triangleleft \cdots \triangleleft G_{1} \triangleleft G_{0}=G
$$

Temos

$$
\{1\}=G_{m} \cap H \triangleleft \cdots \triangleleft G_{1} \cap H \triangleleft G_{0} \cap H=H,
$$

que não é, necessariamente, uma série de composição. Porém, pelos resultados anteriores, basta verificar que os fatores $\left(G_{i} \cap H\right) /\left(G_{i+1} \cap H\right)$ são subgrupos de $\mathfrak{S}_{k}$. Mas $\left(G_{i} \cap H\right) /\left(G_{i+1} \cap H\right)$ é um subgrupo de $G_{i} / G_{i+1}$, e este, por sua vez, é um subgrupo de $\mathfrak{S}_{k}$ por hipótese, o que encerra a demonstração.

Finalmente, podemos mostrar que $\operatorname{Aut}_{e}\left(X_{r}\right) \in \Gamma_{t-1}$. Inicialmente, temos que ker $\pi_{r}$ é o produto direto de grupos $\mathfrak{S}_{f^{-1}(a)}$ e como o número de filhos é $\# f^{-1}(a) \leq t-1$, temos que ker $\pi_{r} \in \Gamma_{t-1}$. Por fim, note que podemos supor, por hipótese de indução, que $\operatorname{Aut}_{e}\left(X_{r}\right) \in \Gamma_{t-1}$. Como $\operatorname{Aut}_{e}\left(X_{r+1}\right) / \operatorname{ker} \pi_{r}$ é isomorfo à imagem de $\pi_{r}$, que é um subgrupo de $\operatorname{Aut}_{e}\left(X_{r}\right)$, temos que $\operatorname{Aut}_{e}\left(X_{r+1}\right) / \operatorname{ker} \pi_{r}$ está em $\Gamma_{k}$, assim como ker $\pi_{r}, \operatorname{logo} \operatorname{Aut}_{e}\left(X_{r+1}\right)$ também está e o resultado segue.

Resumindo, testar isomorfismo de grafos cujos vértices têm grau limitado é polinomialmente equivalente ao seguinte 
Problema Seja $\Omega$ um conjunto colorido. Dado um conjunto de geradores para um subgrupo $G \in \Gamma_{k}$ de $\mathfrak{S}_{\Omega}$, determinar um conjunto de geradores para o subgrupo das permutações que preservam cores.

O algoritmo de Luks resolve este último problema em tempo polinomial no tamanho de $\Omega$. Antes de descrever o algoritmo, precisaremos ainda de mais alguns resultados. É o que veremos a seguir.

\subsection{Primitividade}

Dado um grupo de permutações $G$ agindo sobre um conjunto $\Omega$, podemos particionar $\Omega$ em órbitas $\mathcal{O}_{i}$ e estudar os grupos transitivos de $\mathfrak{S}_{\mathcal{O}_{i}}$ obtidos restringindo-se a ação de $G$ a cada órbita. Como os graus destes grupos são menores, espera-se que o estudo destes seja mais simples ou, no que tange a algoritmos, que o tempo gasto para resolver um determinado problema nestes grupos seja menor do que no grupo original $G$.

Mas o que fazer se o grupo $G$ já é transitivo? Se o grupo for imprimitivo, é possível ainda quebrar o problema em pedaços menores, observando que o grupo $G$ induz uma permutação nos blocos de um sistema de imprimitividade. Os grupos primitivos representam, por assim dizer, o "gargalo" deste procedimento recursivo. Por outro lado, a condição de primitividade pode ser usada a nosso favor, já que ela implica diversas propriedades interessantes. Comecemos pelo seguinte

Teorema 3.2 Seja $G \subset \mathfrak{S}_{\Omega}$ um grupo de permutações. As órbitas de um subgrupo normal $N \triangleleft G$ formam um sistema de imprimitividade para $G$. Em particular, subgrupos normais de grupos primitivos são transitivos.

Demonstração Seja $\left\{B_{1}, B_{2}, \ldots, B_{r}\right\}$ a partição de $\Omega$ em órbitas de $N$. Suponha que, para algum $\sigma \in G, \sigma\left(B_{i}\right) \cap B_{i} \neq \emptyset$ e seja $x \in \sigma\left(B_{i}\right) \cap B_{i}$. Como $B_{i}=N(x)$, existe $\gamma \in N$ tal que $\sigma \gamma(x)=x \Longleftrightarrow \gamma(x)=\sigma^{-1}(x)$. Assim,

$$
\begin{aligned}
\sigma\left(B_{i}\right) & =\{\sigma \tau(x) \mid \tau \in N\}=\{\sigma \tau \gamma(x) \mid \tau \in N\} \\
& =\left\{\sigma \tau \sigma^{-1}(x) \mid \tau \in N\right\}=N(x)=B_{i},
\end{aligned}
$$

o que conclui a demonstração. 
O seguinte teorema mostra que é interessante trabalhar com $p$-grupos.

Teorema 3.3 Seja $P$ um p-grupo transitivo, subgrupo de $\mathfrak{S}_{\Omega}$. Se \#P> $p$, então $P$ é imprimitivo e qualquer sistema de imprimitividade minimal consiste exatamente de $p$ blocos. Além disso, o subgrupo que estabiliza todos os blocos tem indice $p$ em $P$.

Demonstração Fixe um elemento $x \in \Omega$ e seja $H=\operatorname{Stab}(x)$. Se $P$ fosse primitivo, $H$ seria maximal em $P$, logo $[P: H]=p$ pelo teorema de Sylow. Assim, $\# \Omega=[P: H]=p, \operatorname{logo} \# P \mid p$ !. Como $P$ é um $p$-grupo, devemos ter $\# P=p$, o que contradiz o enunciado. Logo $P$ é imprimitivo. Um sistema de imprimitividade minimal corresponde a classes laterais de um subgrupo $K \supset H$ maximal. Como já vimos, $K$ tem índice $p$ e, além disso, como $P$ é $p$-grupo, $K$ é normal. Portanto, sendo $\sigma K$ é uma classe lateral, $K \sigma K=\left(\sigma K \sigma^{-1}\right) \sigma K=\sigma K$, isto é, $K$ estabiliza todos os blocos.

O próximo resultado é central para este capítulo, já que o algoritmo de Luks reduz o problema de um grupo em $\Gamma_{k}$ para um $p$-subgrupo de Sylow suficientemente grande, a fim de aplicar um procedimento recursivo baseado nas idéias discutidas acima. A demonstração será dada no fim do capítulo, após desenvolvermos toda a teoria necessária.

Teorema 3.4 (Resultado fundamental) Existe uma constante $c(k)$ tal que, se $G \in \Gamma_{k}$ é um grupo primitivo de grau n, então, para algum primo $p$, $G$ possui um p-subgrupo de Sylow cujo índice é menor ou igual a $n^{c(k)}$.

\subsection{O algoritmo de Luks}

Podemos agora descrever o algoritmo de Luks [Luk], que resolve o problema de calcular automorfismos de um conjunto colorido.

Seja $\Omega$ um conjunto colorido com $n$ elementos. O número de cores e sua distribuição é irrelevante. Para $a, b \in \Omega$, denotamos a relação " $a$ tem mesma cor que $b "$ por $a \sim b$. Seja $B \subset \Omega$ e $K \subset \mathfrak{S}_{\Omega}$. Definimos

$$
\mathfrak{C}_{B}(K)=\{\sigma \in K \mid \forall b \in B, \sigma(b) \sim b\}
$$

Dados geradores para um subgrupo $G \in \Gamma_{k}$ de $\mathfrak{S}_{\Omega}$ que estabiliza $B$ (isto é, $g(b) \in B$ para todo $g \in G$ e $b \in B$ ) e uma permutação $\sigma \in \mathfrak{S}_{\Omega}$ vamos 
descrever um algoritmo que calcula $\mathfrak{C}_{B}(\sigma G)$. Nosso problema corresponde obviamente ao caso particular em que $B=\Omega$ e $\sigma=1$.

Como $\mathfrak{C}_{B}(\sigma G)$ não é necessariamente um subgrupo de $\mathfrak{S}_{\Omega}$, não está muito claro o que significa "calcular $\mathfrak{C}_{B}(\sigma G)$ ". Entretanto, se $\mathfrak{C}_{B}(\sigma G) \neq \emptyset$, então mostraremos que ele é uma classe lateral à esquerda de $\mathfrak{C}_{B}(G)$ (este sim um grupo), logo podemos fornecer $\mathfrak{C}_{B}(\sigma G)$ através de geradores de $\mathfrak{C}_{B}(G)$ e mais um representante da classe lateral.

De fato, fixe $\pi \in \mathfrak{C}_{B}(\sigma G)$ e seja $\tau \in \mathfrak{C}_{B}(\sigma G)$. Então, para todo $b \in B$, $\tau(b) \sim b \sim \pi(b)$, logo $\pi^{-1} \tau(b) \sim b$. Como $\tau, \pi$ estão na mesma classe lateral $\sigma G$, temos que $\pi^{-1} \tau \in G$, isto é, $\pi^{-1} \tau \in \mathfrak{C}_{B}(G) \Longleftrightarrow \tau \in \pi \mathfrak{C}_{B}(G)$. Por outro lado, para todo $\rho \in \mathfrak{C}_{B}(G)$ e qualquer $b \in B, \rho(b) \sim b \Rightarrow \pi \rho(b) \sim$ $\pi(b) \Rightarrow \pi \rho(b) \sim b$. Portanto $\mathfrak{C}_{B}(\sigma G)=\pi \mathfrak{C}_{B}(G)$, o que encerra a prova.

Temos ainda as seguintes propriedades, que são imediatas a partir da definição: para $K, K^{\prime} \subset \mathfrak{S}_{\Omega}$ e $B, B^{\prime} \subset \Omega$,

- $\mathfrak{C}_{B}\left(K \cup K^{\prime}\right)=\mathfrak{C}_{B}(K) \cup \mathfrak{C}_{B}\left(K^{\prime}\right)$

- $\mathfrak{C}_{B \cup B^{\prime}}(K)=\mathfrak{C}_{B^{\prime}}\left(\mathfrak{C}_{B}(K)\right)$

Vamos ao algoritmo propriamente dito. Se $B$ consiste de um único elemento $b$, temos

$$
\mathfrak{C}_{B}(\sigma G)= \begin{cases}\emptyset & \text { se } \sigma(b) \nsim b \\ \sigma G & \text { se } \sigma(b) \sim b\end{cases}
$$

Se $G$ é intransitivo, podemos determinar suas órbitas $B_{i}, 1 \leq i \leq r$, em tempo polinomial. Basta então aplicar várias vezes o procedimento:

$$
\mathfrak{C}_{B}(\sigma G)=\mathfrak{C}_{B_{1}}\left(\mathfrak{C}_{B_{2}}\left(\ldots \mathfrak{C}_{B_{r}}(\sigma G)\right)\right)
$$

Caso contrário, $G$ é transitivo e \# $B>1$. Se $G$ for imprimitivo, podemos encontrar, em tempo polinomial, um sistema minimal de imprimitividade com $m$ blocos:

$$
B=B_{1} \cup B_{2} \cup \cdots \cup B_{m}
$$

Se $G$ for primitivo, escrevemos a partição acima de modo que cada $B_{i}$ contenha exatamente um elemento de $B$. Sendo $K$ o subgrupo de $G$ que estabiliza todos os blocos, devido à minimalidade do sistema, temos que $G / K$ age primitivamente permutando os $m$ blocos $B_{i}$ 's. Logo $G / K \subset \mathfrak{S}_{m}$ e $G / K \in \Gamma_{k}$ e, portanto, o teorema fundamental garante a existência de um subgrupo $P \supset K$ de $G$ tal que $[G: P] \leq m^{c(k)}$ e $P / K$ age como um $p$-grupo em $\mathcal{B}=\left\{B_{1}, \ldots, B_{m}\right\}$. Tal subgrupo pode ser computado modificando-se, por 
exemplo, o algoritmo que constrói um subgrupo de Sylow: basta alterar os testes nas filtragens de modo a considerar a ação de $G$ sobre $\mathcal{B}$, como visto no final do capítulo anterior.

De posse de $P$, decompomos $G$ em classes laterais:

$$
G=\bigcup \tau_{i} P, \quad \mathfrak{C}_{B}(\sigma G)=\bigcup \mathfrak{C}_{B}\left(\sigma \tau_{i} P\right)
$$

Agora, como $P$ age como $p$-grupo sobre $\mathcal{B}$, é conveniente trabalhar com seus blocos. Assim, estendemos um pouco nossa definição: se $\mathcal{B}=\left\{B_{1}, \ldots, B_{m}\right\}$,

$$
\hat{\mathfrak{C}}_{\mathcal{B}}(\sigma P)=\mathfrak{C}_{B}(\sigma P),
$$

em que $B=B_{1} \cup \cdots \cup B_{m}$.

O cálculo procede como antes, só que agora mantemos a integridade dos blocos o maior tempo possível:

1. se $\mathcal{B}$ consiste de um único bloco $B, \hat{\mathfrak{C}}_{\mathcal{B}}(\sigma P)=\mathfrak{C}_{B}(\sigma P)$.

2. se $\mathcal{B}=\mathcal{B}^{\prime} \cup \mathcal{B}^{\prime \prime}$ é a união disjunta de duas coleções estabilizadas por $P$,

$$
\hat{\mathfrak{C}}_{\mathcal{B}}(\sigma P)=\hat{\mathfrak{C}}_{\mathcal{B}^{\prime}}\left(\hat{\mathfrak{C}}_{\mathcal{B}^{\prime \prime}}(\sigma P)\right)
$$

3. se $P$ é transitivo em $\mathcal{B}$ e $\mathcal{B}$ possui mais de um bloco, particionamos $\mathcal{B}$ em um sistema minimal de imprimitividade:

$$
\mathcal{B}=\mathcal{B}_{1} \cup \cdots \cup \mathcal{B}_{p}
$$

Aqui, uma observação. Se $\#(P / K)=p$, então $P / K$ age primitivamente sobre os blocos de $\mathcal{B}$. Entretanto, consideraremos a partição trivial de $\mathcal{B}$ em conjuntos unitários como um "sistema de imprimitividade" para $P / K$.

De posse deste sistema de imprimitividade minimal, determinamos o subgrupo $P^{\prime}$ que estabiliza todas as subcoleções $\mathcal{B}_{i}$. Decompomos $P$ em classes laterais e aplicamos novamente o algoritmo:

$$
P=\bigcup \tau_{i} P^{\prime}, \quad \hat{\mathfrak{C}}_{\mathcal{B}}(\sigma P)=\bigcup \hat{\mathfrak{C}}_{\mathcal{B}}\left(\sigma \tau_{i} P^{\prime}\right)
$$

A vantagem de trabalharmos com $P$ agora fica evidente: podemos quebrar o conjunto $\mathcal{B}$ em partes $P^{\prime}$-estáveis e resolver problemas menores:

$$
\hat{\mathfrak{C}}_{\mathcal{B}}\left(\sigma \tau_{i} P^{\prime}\right)=\hat{\mathfrak{C}}_{\mathcal{B}_{1}}\left(\hat{\mathfrak{C}}_{\mathcal{B}_{2}}\left(\ldots \hat{\mathfrak{C}}_{\mathcal{B}_{p}}\left(\sigma \tau_{i} P^{\prime}\right)\right)\right)
$$


Vamos analisar a complexidade deste algoritmo. Após o passo 3, temos que o problema para um conjunto de tamanho $n$ se reduz a $p^{2}$ problemas de tamanho $n / p$. Aplicando o procedimento acima até obtermos um único bloco (passo 1), teremos, ao final, no máximo $m^{2}$ subproblemas de tamanho $n / m$. Como $[G: P] \leq m^{c(k)}$, reduzimos o problema original em não mais do que $m^{c(k)+2}$ problemas menores, de tamanho $n / m$.

Todas as operações envolvidas neste processo são polinomiais em $n$, tais como o particionamento de $\mathcal{B}$ em blocos, a determinação dos subgrupos $P$ e $P^{\prime}$, etc. Logo o tempo $T(n)$ gasto pelo algoritmo satisfaz

$$
T(n)=m^{c(k)+2} T(n / m)+O\left(n^{l}\right)
$$

para $m \geq 2$ e algum $l \geq 0$. A solução desta recursão é polinomial em $n$. Com isto, terminamos a análise do algoritmo de Luks.

\subsection{Socle}

Definimos o socle de um grupo finito como o subgrupo gerado por todos os seus subgrupos normais minimais. O socle é uma importante ferramenta no estudo de grupos primitivos e, como veremos em seguida, terá um papel crucial na demonstração da existência do subgrupo de Sylow de índice polinomial. Mas antes de tratar do socle propriamente dito, é melhor aprendermos um pouco mais sobre seus componentes, os subgrupos normais minimais.

Teorema 3.5 Num grupo, quaisquer dois subgrupos normais minimais comutam.

Demonstração Sejam $G$ e $H$ dois subgrupos normais minimais. Temos que $G \cap H$ é um subgrupo normal, pois para qualquer elemento $x$ do grupo, $x^{-1}(G \cap H) x \subset x^{-1} G x=G$ e, analogamente, $x^{-1}(G \cap H) x \subset H$, ou seja, $x^{-1}(G \cap H) x \subset G \cap H$. Como $G \cap H \subsetneq G$ e $G$ é minimal, temos que $G \cap H=\{1\}$. Assim, sendo $g \in G$ e $h \in H$, temos

$$
\begin{aligned}
& g h g^{-1} h^{-1}=\left(g h g^{-1}\right) h^{-1} \in H \\
& g h g^{-1} h^{-1}=g\left(h g^{-1} h^{-1}\right) \in G
\end{aligned}
$$

Logo $g h g^{-1} h^{-1}=1$, ou seja, $g h=h g$. 
Teorema 3.6 Grupos normais minimais são produtos diretos de grupos simples isomorfos.

Demonstração Seja $N$ um subgrupo normal minimal de $G$. Seja $\operatorname{Aut}(N)$ o grupo de automorfismos de $N$ e considere um subgrupo normal minimal $T$ de $N$. Vamos mostrar que $N$ é o produto direto de alguns dos grupos $\sigma(T)$, $\sigma \in \operatorname{Aut}(N)$.

Seja $M$ o maior subgrupo de $N$ que é produto direto de grupos da forma $\sigma(T)$. Como cada $\sigma(T)$ é normal em $N$, temos que $M$ é normal em $N$ também e, além disso, contém todos os $\sigma(T)$. De fato, se existisse um $\tau(T) \not \subset M$, teríamos que $\tau(T) \cap M \subsetneq \tau(T)$ e, pela minimalidade de $\tau(T), \tau(T) \cap M=\{1\}$. Logo $M$ e $\tau(T)$ comutariam e $M \tau(T)$ seria um subgrupo maior do que $M$ e que é produto direto de $\sigma(T)$, o que é absurdo. Assim, $M$ contém todo grupo da forma $\sigma(T)$ e, portanto, $\sigma(M)=M$ para todo $\sigma \in \operatorname{Aut}(N)$. Em particular, $g^{-1} M g=M$ para todo $g \in G$. Logo, devido à minimalidade de $N$, temos que $M=N$ e, portanto, $N$ é produto direto de grupos isomorfos a $T$.

Para terminar a demonstração, temos que verificar que $T$ é simples. Mas isto é fácil: como $N$ é produto direto de grupos da forma $\sigma(T)$, qualquer subgrupo normal de $T$ é também subgrupo normal de $N$. Se $T$ não fosse simples, teríamos uma contradição, já que $T$ foi escolhido como um subgrupo normal minimal de $N$.

Precisaremos ainda do seguinte resultado.

Teorema 3.7 Sejam $H$ e $H^{\prime}$ dois subgrupos transitivos de $\mathfrak{S}_{\Omega}$ que comutam

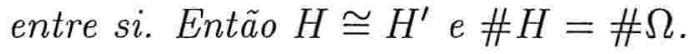

Demonstração Fixe $x \in \Omega$. Para cada $h \in H$, existe um $h^{\prime} \in H^{\prime}$ tal que $h(x)=h^{\prime}(x)$, devido à transitividade de $H^{\prime}$. Suponha agora que $h^{\prime}(x)=$ $h^{\prime \prime}(x)$ para $h^{\prime}, h^{\prime \prime} \in H^{\prime}$. Como $H$ e $H^{\prime}$ comutam, temos, para todo $g \in H$,

$$
h^{\prime}(x)=h^{\prime \prime}(x) \Rightarrow g h^{\prime}(x)=g h^{\prime \prime}(x) \Longleftrightarrow h^{\prime}(g(x))=h^{\prime \prime}(g(x))
$$

Mas $H$ é transitivo, logo $h^{\prime}(y)=h^{\prime \prime}(y)$ para todo $y \in \Omega$ e temos que $h^{\prime}=h^{\prime \prime}$.

Assim, a cada $h \in H$ podemos associar o único elemento $h^{\prime} \in H^{\prime}$ tal que $h(x)=h^{\prime}(x)$. Como esta associação é simétrica com relação a $H$ e $H^{\prime}$, temos que ela estabelece uma bijeção entre estes dois grupos. Esta bijeção não é 
exatamente um homomorfismo, pois dados $g, h \in H$ e $g^{\prime}, h^{\prime} \in H^{\prime}$ tais que $g(x)=g^{\prime}(x)$ e $h(x)=h^{\prime}(x)$, temos

$$
h(x)=h^{\prime}(x) \Rightarrow g h(x)=g h^{\prime}(x)=h^{\prime} g(x)=h^{\prime} g^{\prime}(x)
$$

Logo $g h$ está associado a $h^{\prime} g^{\prime}$. Entretanto podemos definir $\phi(h)=h^{\prime-1}$ e desta forma obter o isomorfismo procurado, já que

$$
\phi(g h)=\left(h^{\prime} g^{\prime}\right)^{-1}=g^{\prime-1} h^{\prime-1}=\phi(g) \phi(h)
$$

Para terminar, devemos mostrar que $\# H=\# \Omega$. Como

$$
\# H=\# \operatorname{Stab}(x) \cdot \# H(x)=\# \operatorname{Stab}(x) \cdot \# \Omega,
$$

basta mostrar que se $h(x)=x$ para $h \in H$, então $h=1$. Neste caso, porém, temos $\phi(h)=1 \Longleftrightarrow h=1$, o que encerra a demonstração.

Vamos agora examinar mais detalhadamente o caso que nos interessa: o socle de um grupo primitivo. No que segue, $G$ é um grupo primitivo, subgrupo de $\mathfrak{S}_{\Omega}$, e $N$ é um subgrupo normal minimal. Suponha que $G$ possua outro subgrupo normal minimal $N^{\prime}$. Então, pelos teoremas acima, sabemos que $N$ e $N^{\prime}$ são transitivos e comutam. Assim, temos

Teorema $3.8 N \cong N^{\prime}$

Por outro lado, $N^{\prime} \subset \mathbf{Z}_{G}(N)$. Logo $\mathbf{Z}_{G}(N)$ é transitivo também e comuta com $N$. Portanto $\# \mathbf{Z}_{G}(N)=\# \Omega=\# N^{\prime}$, isto é, $N^{\prime}=\mathbf{Z}_{G}(N)$. Resumindo, temos

Teorema $3.9 N^{\prime}$ é precisamente o centralizador $\mathbf{Z}_{G}(N)$ de $N$ em $G$ (e viceversa).

A conclusão que se pode tirar destas duas proposições é que $G$ não possui outros subgrupos normais minimais além de $N$ e $N^{\prime}$, já que qualquer outro subgrupo normal minimal deveria estar em $\mathbf{Z}_{G}(N)$. Além disso, do fato de que qualquer subgrupo normal minimal é produto direto de subgrupos simples, obtemos o

Teorema 3.10 $O$ socle de um grupo primitivo é o produto direto de um ou dois subgrupos normais minimais. Em ambos os casos, o socle é produto de grupos simples isomorfos. 
No caso em que o socle é abeliano, temos $N \subset \mathrm{Z}_{G}(N)$, e um argumento similar aos anteriores permite concluir que $N=\mathrm{Z}_{G}(N)$. Desta forma, não há outros subgrupos normais minimais e $N$ é o socle de $G$. Como um grupo abeliano simples é isomorfo a $\mathbb{Z}_{p}$, para algum primo $p$, temos $N \cong \mathbb{Z}_{p} \oplus$ $\mathbb{Z}_{p} \oplus \cdots \oplus \mathbb{Z}_{p}$ ( $d$ vezes). Na verdade, podemos até obter uma descrição mais completa do grupo $G$.

Teorema 3.11 Se o socle de um grupo primitivo $G$ é abeliano, então o grau de $G$ é uma potência $p^{d}$ de um certo primo $p$ e $G$ é isomorfo a um subgrupo de $A G L(d, p)$, o grupo das transformações afins de $\mathbb{Z}_{p}^{d}$.

O grupo $A G L(d, p)$ é gerado pelo grupo das transformações lineares inversíveis de $\mathbb{Z}_{p}^{d}$, o $G L(d, p)$, e pelo grupo das translações de $\mathbb{Z}_{p}^{d}$.

Demonstração Como $N \cong \mathbb{Z}_{p} \oplus \mathbb{Z}_{p} \oplus \cdots \oplus \mathbb{Z}_{p}$ ( $d$ vezes) é transitivo e comuta consigo mesmo, temos $\# \Omega=\# N=p^{d}$, ou seja, o grau de $G$ é $p^{d}$.

Vamos construir agora uma injeção de $G$ em $A G L(d, p)$. Observemos inicialmente que temos um homomorfismo de $G$ em $\operatorname{Aut}(N)$, que leva $g \in G$ na "anti-conjugação" $A_{g} \in \operatorname{Aut}(N)$ dada por $A_{g}(z)=g z g^{-1}$. Note que o kernel deste homomorfismo é $\mathbf{Z}_{G}(N)=N$, de modo que temos uma injeção de $G / N$ em $\operatorname{Aut}(N)$.

Fixemos um isomorfismo $i: N \rightarrow \mathbb{Z}_{p} \oplus \cdots \oplus \mathbb{Z}_{p}$. Compondo um automorfismo $A_{g}$ de $N$ com o isomorfismo $i$, obtemos um automorfismo $i \circ A_{g} \circ i^{-1}$ de $\mathbb{Z}_{p} \oplus \cdots \oplus \mathbb{Z}_{p}$. Porém, os automorfismos de $\mathbb{Z}_{p} \oplus \cdots \oplus \mathbb{Z}_{p}$ são exatamente as transformações lineares inversíveis de $\mathbb{Z}_{p} \oplus \cdots \oplus \mathbb{Z}_{p}$, visto como o espaço vetorial $\mathbb{Z}_{p}^{d}$ sobre $\mathbb{Z}_{p}$ : é claro que qualquer transformação linear inversível é um automorfismo; reciprocamente, devemos verificar que, para qualquer automorfismo $F, F(x+y)=F(x)+F(y)$ e $F(c x)=c F(x), x, y \in \mathbb{Z}_{p} \oplus \cdots \oplus \mathbb{Z}_{p}$ e $c \in \mathbb{Z}_{p}$. Porém, a primeira condição segue diretamente do fato de que $F$ é automorfismo e a segunda segue da primeira e da relação

$$
F(c x)=F(x+\cdots+x)=F(x)+\cdots+F(x)=c F(x)
$$

Assim, temos uma injeção de $G / N$ em $G L(d, p)$, dada por $g N \rightarrow i \circ A_{g} \circ i^{-1}$.

Finalmente, seja $\omega \in \Omega$ um elemento fixado e, para cada $g \in G$, seja $n_{g} \in N$ o único elemento tal que $g(\omega)=n_{g}(\omega)$. Definimos $\phi(g) \in A G L(d, p)$ como a composição de $i \circ A_{g} \circ i^{-1} \in G L(d, p)$ e da translação por $i\left(n_{g}\right)$, isto é, o elemento de $A G L(d, p)$ tal que $\phi(g)(x)=i \circ A_{g} \circ i^{-1}(x)+i\left(n_{g}\right)=$ $i\left(A_{g} \circ i^{-1}(x) n_{g}\right)$, para todo $x \in \mathbb{Z}_{p}^{d}$. 
Sejam $g, h \in G$. Temos, para todo $x \in \mathbb{Z}_{p}^{d}$,

$$
\begin{aligned}
\phi(g) \phi(h)(x) & =\phi(g)\left(i\left(A_{h} \circ i^{-1}(x) n_{h}\right)\right) \\
& =i\left(A_{g}\left(A_{h} \circ i^{-1}(x) n_{h}\right) n_{g}\right) \\
& =i\left(A_{g h} \circ i^{-1}(x) A_{g}\left(n_{h}\right) n_{g}\right) \\
& =i \circ A_{g h} \circ i^{-1}(x)+i\left(A_{g}\left(n_{h}\right) n_{g}\right)
\end{aligned}
$$

Porém, $A_{g}\left(n_{h}\right) n_{g} \in N$ e $A_{g}\left(n_{h}\right) n_{g}(\omega)=A_{g}\left(n_{h}\right) g(\omega)=\left(g n_{h} g^{-1}\right) g(\omega)=$ $g n_{h}(\omega)=g h(\omega)$, isto é, $n_{g h}$ é precisamente $A_{g}\left(n_{h}\right) n_{g}$. Logo

$$
\phi(g) \phi(h)(x)=i \circ A_{g h} \circ i^{-1}(x)+i\left(n_{g h}\right)=\phi(g h)(x)
$$

Conseqüentemente, $\phi$ é um homomorfismo de $G$ em $A G L(d, p)$. Se $\phi(g)$ é a identidade, então, como ele deve levar $0 \in \mathbb{Z}_{p}^{d}$ em 0 , concluímos que $i\left(n_{g}\right)=0 \Longleftrightarrow n_{g}=1 \Longleftrightarrow g(\omega)=\omega$. Por outro lado, $i \circ A_{g} \circ i^{-1} \in G L(d, p)$ também é a identidade, ou seja, $g$ está no kernel de $g \rightarrow i \circ A_{g} \circ i^{-1}$, que é $N$. Portanto $g=1$, em outras palavras, $\phi$ é injetora, completando a demonstração.

Precisaremos estimar a ordem de $G$, o que pode ser feito utilizando o resultado anterior e conhecendo-se a ordem de $A G L(d, p)$.

Teorema 3.12 A ordem de $A G L(d, p)$ é

$$
\# A G L(d, p)=p^{\frac{d(d+1)}{2}}(p-1)\left(p^{2}-1\right)\left(p^{3}-1\right) \cdots\left(p^{d}-1\right)
$$

Demonstração Uma transformação afim pode ser escrita de maneira única como a composição de um elemento em $G L(d, p)$ e uma translação. Logo a. ordem procurada é o produto das ordens destes dois grupos.

Uma translação é dada por um vetor em $\mathbb{Z}_{p}^{d}$, logo o grupo das translações é isomorfo a $\mathbb{Z}_{p} \oplus \mathbb{Z}_{p} \oplus \cdots \oplus \mathbb{Z}_{p}$ ( $d$ vezes), que tem $p^{d}$ elementos.

Um elemento de $G L(d, p)$ pode ser dado por uma matriz quadrada de ordem $d$ e determinante não nulo. $\mathrm{O}$ número de tais matrizes pode ser facilmente obtido da seguinte forma: há $p^{d}-1$ escolhas para a primeira coluna, que não pode ser nula; para a segunda, podemos escolher qualquer vetor que não seja múltiplo da primeira, ou seja, há $p^{d}-p$ escolhas para a segunda coluna. Em geral, para a $n$-ésima coluna, podemos escolher qualquer 
vetor que não seja combinação linear das $n-1$ anteriores, isto é, há $p^{d}-p^{n-1}$ escolhas para esta coluna. Assim,

$$
\begin{aligned}
\# G L(d, p) & =\left(p^{d}-1\right)\left(p^{d}-p\right)\left(p^{d}-p^{2}\right) \cdots\left(p^{d}-p^{d-1}\right) \\
& =p^{\frac{d(d-1)}{2}}\left(p^{d}-1\right)\left(p^{d-1}-1\right)\left(p^{d-2}-1\right) \cdots(p-1)
\end{aligned}
$$

Multiplicando as ordens, o resultado segue.

\subsection{Demonstrando o resultado fundamental}

Estamos prontos para demonstrar a existência do subgrupo de Sylow de índice polinomial. Dividiremos a prova em dois casos, conforme o socle seja ou não abeliano.

\subsubsection{O socle é abeliano}

Neste caso, sabemos que $G \subset A G L(d, p)$ para algum primo $p$ e $d=\log _{p} n$ (lembre-se de que $n$ é o grau de $G$ ). Vamos mostrar que qualquer $p$-subgrupo de Sylow de $G$ tem a propriedade desejada, calculando seu índice. Para isto, precisaremos do seguinte resultado.

Teorema 3.13 Sejam p e q primos distintos. Então existe uma constante $\alpha(p, q)$ tal que o maior expoente $x$ para o qual $q^{x}$ divide \#AGL $(d, p)$ é menor do que $d \alpha(p, q)$.

Demonstração Lembramos que

$$
\# A G L(d, p)=p^{\frac{d(d+1)}{2}}(p-1)\left(p^{2}-1\right)\left(p^{3}-1\right) \cdots\left(p^{d}-1\right)
$$

Assim, para determinar o maior expoente $x$ para o qual $q^{x}$ divide a expressão acima, precisamos determinar antes a contribuição de cada fator $p^{y}-1$. Seja então $n(y)$ o expoente da maior potência de $q$ que divide $p^{y}-1$, isto é,

$$
p^{y}-1=a q^{n(y)}, \quad q \nmid a
$$


Suponha que $n(y) \geq 1$, isto é, que $q$ realmente divide $p^{y}-1$. Vamos ver o que ocorre quando multiplicamos $y$ por $z$. Temos

$$
\begin{aligned}
p^{y z} & =\left(1+a q^{n(y)}\right)^{z} \\
& =\left(\begin{array}{l}
z \\
0
\end{array}\right)+\left(\begin{array}{l}
z \\
1
\end{array}\right) a q^{n(y)}+\left(\begin{array}{c}
z \\
2
\end{array}\right)\left(a q^{n(y)}\right)^{2}+\cdots \\
& \equiv 1+a z q^{n(y)}+\frac{a^{2} z(z-1)}{2} \cdot q^{2 n(y)}\left(\bmod q^{3 n(y)}\right),
\end{aligned}
$$

ou seja,

$$
p^{y z}-1 \equiv q^{n(y)}\left(a z+\frac{a^{2} z(z-1)}{2} \cdot q^{n(y)}\right) \quad\left(\bmod q^{3 n(y)}\right)
$$

e $3 n(y)>n(y)+1$. Portanto

- $n(y z)=n(y)$ se $q \nmid z$

- $n(y q)=n(y)+1$ se $q \neq 2$ ou $n(y) \geq 2$

- $n(y q) \geq n(y)+2$ se $q=2$

Seja $r$ a ordem de $p \bmod q$. Então $n(y)>0 \Longleftrightarrow r \mid y$. Temos ainda que, se $q \neq 2$, então $n\left(r q^{u}\right)=n(r)+u$. Por outro lado, se $q=2, r=1$. Neste caso, se $n(1) \geq 2$, temos $n\left(2^{u}\right)=n(1)+u$, como antes. Porém, se $n(1)=$ $1 \Longleftrightarrow p \equiv 3 \quad(\bmod 4)$, então $n(2) \geq 3$ e $n\left(2^{u}\right)=n\left(2 \cdot 2^{u-1}\right)=n(2)+u-1$.

Seja $q$ ímpar ou $n(r) \geq 2$. Para determinar o expoente da maior potência de $q$ que divide $|A G L(d, p)|$, basta determinar a quantidade de múltiplos de $r$ : cada um destes contribui com $n(r)$ no expoente. Dentre estes múltiplos, aqueles que também são múltiplos de $r q$ contribuem com mais 1 , dentre estes últimos, aqueles múltiplos de $r q^{2}$ contribuem com mais 1 e assim por diante. Logo o expoente procurado é

$$
\begin{aligned}
x & =n(r)\left\lfloor\frac{d}{r}\right\rfloor+\left\lfloor\frac{d}{r q}\right\rfloor+\left\lfloor\frac{d}{r q^{2}}\right\rfloor+\left\lfloor\frac{d}{r q^{3}}\right\rfloor+\cdots \\
& <n(r) \frac{d}{r}+\frac{d}{r q}+\frac{d}{r q^{2}}+\frac{d}{r q^{3}}+\cdots \\
& =d \cdot \frac{n(r)+1 /(q-1)}{r}
\end{aligned}
$$


Se $q=2$ e $n(1)=1$, basta modificar o segundo termo:

$$
\begin{aligned}
x & =d+(n(2)-1)\left\lfloor\frac{d}{2}\right\rfloor+\left\lfloor\frac{d}{2^{2}}\right\rfloor+\left\lfloor\frac{d}{2^{3}}\right\rfloor+\cdots \\
& <n(2) \frac{d}{2}+\frac{d}{2}+\frac{d}{2^{2}}+\frac{d}{2^{3}}+\cdots \\
& =d \cdot \frac{n(2)+2}{2}
\end{aligned}
$$

Em ambos os casos, temos a existência da constante $\alpha(p, q)$ procurada, cujo valor pode ser obtido a partir das fórmulas acima.

De posse deste resultado, é fácil concluir. O índice de um $p$-subgrupo de Sylow de $G$ é dado pelo produto de todas as potências de primos $q$ distintos de $p$ em $|G|$. Temos que se $q^{x}$ é a maior potência de $q$ que divide $|G|$, então

$$
q^{x}<q^{d \alpha(p, q)}=\left(p^{d}\right)^{\alpha(p, q) \log _{p} q}=n^{\alpha(p, q) \log _{p} q}
$$

Portanto, como $G \in \Gamma_{k}$, os fatores primos de $|G|$ não ultrapassam $k$ e, desta forma, o índice do $p$-subgrupo de Sylow é menor ou igual a $n^{c}$, em que

$$
c=\max _{p \leq k} \sum_{q \leq k, q \neq p} \alpha(p, q) \log _{p} q
$$

\subsubsection{O socle não é abeliano}

Começamos com uma estimativa do grau de uma representação de um produto de grupos simples.

Teorema 3.14 Seja $N \subset \mathfrak{S}_{\Omega}$ um grupo de permutações transitivo. Se $N=$ $T_{1} \times T_{2} \times \cdots \times T_{d}$ é produto direto de d grupos simples não abelianos $T_{i}$, então o grau de $N$ é maior ou igual a $5^{d}$.

Demonstração Para $d=1$ o resultado segue do fato de que não há grupos de permutações simples não abelianos de grau menor ou igual a 4 .

Suponha $d>1$. Para um $i$ fixado, as órbitas de $T_{i}$ formam um sistema de imprimitividade para. $N$. De fato, se $\sigma \in N$, podemos escrever $\sigma=t_{1} t_{2} \ldots t_{d}$, $t_{j} \in T_{j}$, logo, para $x \in \Omega$, temos $\sigma T_{i}(x)=T_{i}\left(t_{1} \ldots t_{i-1} t_{i+1} \ldots t_{d}(x)\right)$, isto é, $\sigma$ 
permuta as órbitas de $T_{i}$. Note que, em particular, todas as órbitas possuem o mesmo tamanho.

Podemos supor, sem perda de generalidade, que $T_{1}$ é o fator que possui menores órbitas. Sejam $B_{1}, B_{2}, \ldots B_{m}$ estas órbitas. Seja ainda $K$ o subgrupo de $N$ que estabiliza todas estas órbitas. Temos que $K \triangleleft N$ e, como os $T_{i}$ 's são simples, temos que $K$ é o produto de algum destes fatores, digamos

$$
K=T_{1} \times T_{2} \times \cdots \times T_{s}, \quad s \geq 1
$$

Temos que

$$
N / K \cong T_{s+1} \times T_{s+2} \times \cdots \times T_{d}
$$

age fiel e transitivamente sobre os blocos $B_{i}$, logo por hipótese de indução concluímos que $m \geq 5^{d-s}$. Além disso, o produto de fatores $T_{i}$ de $K$ também age fiel e transitivamente sobre os elementos de $B_{1}$. A transitividade decorre da minimalidade do tamanho das órbitas e do fato de que cada $T_{i}$ em $K$ estabiliza todos os blocos, $B_{1}$ em particular. Para mostrar que a ação é fiel, suponha que um elemento $\sigma$ do produto fixe cada elemento de $B_{1}$. Para cada $j, 2 \leq j \leq m$, escolhemos $\tau_{j} \in T_{s+1} \times \cdots \times T_{d}$ tal que $\tau_{j}\left(B_{1}\right)=B_{j}$. Como $\sigma$ e $\tau_{j}$ comutam, $\sigma$ fixa cada elemento em $B_{j}$ também, logo fixa todos os elementos de $\Omega$ e, portanto, é a identidade.

Temos dois casos. Se $s=1$, temos que $T_{1}$ age fiel e transitivamente sobre $B_{1}, \operatorname{logo}$ por hipótese de indução $\# B_{1} \geq 5$ e $\# \Omega=m \cdot \# B_{1} \geq 5^{d-1} \cdot 5=5^{d}$. Se $s>1$, observe que

$$
K^{\prime \prime}=T_{2} \times T_{3} \times \cdots \times T_{s}
$$

e $T_{1}$ são dois subgrupos que comutam entre si e agem fiel e transitivamente sobre $B_{1}$. Logo $K^{\prime \prime} \cong T_{1}$ e \# $B_{1}=\# T_{1}$. Assim, $s=2$ e \# $B_{1} \geq 60$, logo $\# \Omega=m \cdot \# B_{1} \geq 5^{d-2} \cdot 60>5^{d}$.

Finalmente, podemos concluir a demonstração do teorema fundamental.

Teorema 3.15 Se $G \subset \Gamma_{k}$ é um grupo primitivo de grau $n$ com socle não abeliano, então existe uma constante $c(k)$ tal que $\# G \leq n^{c(k)}$.

Demonstração Sabemos que o socle $N$ é o produto $T_{1} \times \cdots \times T_{d}$ de $d$ grupos simples não abelianos isomorfos. Nossa primeira tarefa será mostrar que $G$ pode ser identificado como subgrupo de $\operatorname{Aut}(N)$. Isto é fácil: temos 
que cada elemento $g \in G$ induz um automorfismo de $N$ por conjugação: $g \rightarrow T_{g}$, em que $T_{g}(x)=g^{-1} x g$ para todo $x \in N$. Se $g$ induz a identidade, então $g$ está em $\mathbf{Z}_{G}(N)$. Porém, como $N$ é o produto de dois subgrupos normais minimais não abelianos e tais que um é o centralizador do outro, temos que $\mathbf{Z}_{G}(N)$ está na intersecção destes dois subgrupos e é, portanto, necessariamente trivial.

Lembrando que $T_{i}$ 's são subgrupos normais minimais de $N$, temos que um automorfismo de $N$ consiste de uma permutação destes fatores seguida da aplicação de um automorfismo de $T_{1} \cong T_{i}$ em cada fator. Isto corresponde ao produto trançado (wreath product)

$$
\operatorname{Aut}(N) \cong \operatorname{Aut}\left(T_{1}\right)>\mathfrak{S}_{d}
$$

Como \#G só é divisível por primos menores ou iguais a $k$, a projeção de $G$ em $\mathfrak{S}_{d}$ tem ordem menor ou igual a $a^{d}$. Aqui $a$ só depende de $k$. Assim,

$$
\# G \leq\left(\# \operatorname{Aut}\left(T_{1}\right)\right)^{d} \cdot a^{d}
$$

Além disso, como $G \in \Gamma_{k}$, temos que $T_{1} \subset \mathfrak{S}_{k}$, logo \# Aut( $\left.T_{1}\right)$ é limitado. Assim, $\# G \leq b^{d}$ para alguma constante $b$ que só depende de $k$. Aplicando o teorema anterior, sabemos que $n \geq 5^{d} \Longleftrightarrow d \leq \log _{5} n$ e, portanto,

$$
\# G \leq n^{\log _{5} b}
$$




\section{Capítulo 4}

\section{Automorfismos e Especializações}

Neste último capítulo, apresentaremos uma abordagem alternativa para o problema de decidir se o grupo de automorfismos de um grafo é ou não trivial. Embora os resultados sejam poucos e incipientes, talvez possam se revelar úteis no estudo de grupos de automorfismo futuramente.

Utilizaremos a correspondência estabelecida por Galois para reformular o problema de determinar o grupo de automorfismos de um grafo. Esta nova formulação servirá de ponto de partida para um programa que pretende atacar a questão de certificar a trivialidade do grupo de automorfismos de um grafo em tempo polinomial.

\subsection{Reformulando o problema}

Seja $k$ um corpo. Dado um grafo $X$ com $V(X)=\{1,2, \ldots, n\}$, construímos um polinômio $\phi_{X} \in k[\mathrm{x}]\left(k[\mathrm{x}]\right.$ denota $\left.k\left[x_{1}, \ldots, x_{n}\right]\right)$, associado a $X$, da seguinte maneira:

$$
\phi_{X}\left(x_{1}, \ldots, x_{n}\right)=\sum_{i j \in E(X)} x_{i} x_{j}
$$

Os elementos do grupo simétrico $\mathfrak{S}_{n}$ agem sobre as variáveis $x_{i}$ permutandoas: definimos $\sigma\left(x_{i}\right)=x_{\sigma(i)}$ para todo $\sigma \in \mathfrak{S}_{n}$ e estendemos esta ação para $L=k(\mathrm{x})$ de modo natural. Podemos então identificar o grupo de automorfismos de $X, \operatorname{Aut}(X)$, como o subgrupo das permutações $\sigma \in \mathfrak{S}_{n}$ que fixam $\phi_{X}$, isto é, tais que $\sigma\left(\phi_{X}\right)=\phi_{X}$. 
Considere agora o corpo $K$ das funções simétricas em $x_{1}, \ldots, x_{n}$. Temos que $K$ pode ser gerado sobre $k$ pelos polinômios simétricos elementares $s_{1}, s_{2}, \ldots, s_{n}$, por exemplo. Utilizando uma notação vetorial como acima, escrevemos $K=k(\mathbf{s})$ para denotar $k\left(s_{1}, s_{2}, \ldots, s_{n}\right)$.

Note que a extensão $L / K$ é galoisiana, pois $L$ é o corpo de raízes do polinômio $t(x)=\prod_{1 \leq i \leq n}\left(x-x_{i}\right)$. Temos que $\operatorname{Gal}(L / K)=\mathfrak{S}_{n}$. Além disso, como $\sigma\left(\phi_{X}\right)=\phi_{X}$ se, e somente se, $\sigma$ fixa cada elemento de $K\left(\phi_{X}\right)$, Aut $(X)$ é nada mais nada menos que o subgrupo de $\operatorname{Gal}(L / K)$ que fixa o corpo $K\left(\phi_{X}\right)$, isto é, $\operatorname{Gal}\left(L / K\left(\phi_{X}\right)\right)$. Desta forma, calcular Aut $(X)$ é equivalente a calcular este grupo de Galois.

A fim de obter informações sobre $\operatorname{Aut}(X)$, utilizaremos a idéia de "especialização", isto é, substituiremos as variáveis $x_{i}$ por valores de uma extensão de $k$ mais "trabalhável". Este procedimento se justifica devido ao seguinte

Teorema 4.1 Dado um grafo $X$, existe um polinômio $m_{X}(\mathrm{x}) \in k[\mathrm{x}]$ tal que qualquer especialização $\mathrm{x} \leftarrow \alpha$ satisfazendo $m_{X}(\alpha) \neq 0$ possui a seguinte propriedade: sendo $\overline{s_{i}}=s_{i}(\alpha)$ e $\overline{\phi_{X}}=\phi_{X}(\alpha)$, o grupo de Galois de $k(\alpha)$ sobre $k\left(\overline{\mathrm{s}}, \overline{\phi_{X}}\right)$ é um subgrupo de Aut $(X)$.

Este resultado sugere uma possível abordagem para obter um certificado de trivialidade para o grupo de automorfismos de um grafo. Para isto, vamos descrever algumas propriedades desejáveis para uma família de especializações que nos ajudassem a resolver o problema:

(i) para cada especialização $\mathrm{x} \leftarrow \alpha$ da família, o problema de decidir se $\operatorname{Gal}\left(k(\alpha) / k\left(\overline{\mathbf{s}}, \overline{\phi_{X}}\right)\right.$ ) é trivial (isto é, se $k(\alpha)=k\left(\overline{\mathbf{s}}, \overline{\phi_{X}}\right)$ ) está em NP;

(ii) se $\operatorname{Gal}\left(L / K\left(\phi_{X}\right)\right)$ não for trivial, então existe uma especialização da família para a qual o grupo de Galois associado não é trivial;

(iii) a família tem tamanho polinomial em $|V(X)|$.

Tal família de especializações permitiria testar eficientemente se um dado grafo $X$ tem ou não automorfismo não trivial: bastaria testar, para cada uma das especializações da família, a igualdade de corpos acima descrita; se para alguma especialização ela não ocorrer, então haverá um automorfismo não trivial; caso contrário, teremos um certificado da trivialidade de $\operatorname{Aut}(X)$. Observe ainda que se o problema em (i) estiver em $\mathrm{P}$, concluiríamos que testar isomorfismos de grafos também está em P. 
Poderia se questionar quanto à existência de especializações que satisfaçam (ii) ou de extensões de corpos com a propriedade (i). Para (ii), é possível escolher uma família com uma única especialização em que os $x_{i}$ 's são as raízes de um polinômio irredutível de grau $n$ e grupo de Galois $\mathfrak{S}_{n}$ (a existência deste polinômio é um corolário do Teorema de Irredutibilidade de Hilbert, veja, por exemplo, [Had]). Infelizmente não se sabe responder (i) eficientemente para esta família. Por outro lado, há diversas extensões de grau polinomial em $|V(X)|$ satisfazendo (i), como extensões de corpos finitos, extensões ciclotômicas de $\mathbb{Q}$, etc. Para algoritmos que resolvem esta e questões similares, veja [Coh].

Por simplicidade, podemos escolher os valores $\alpha_{i}$ como raízes de um polinômio com coeficientes em $k$; neste caso, teremos que $\overline{s_{i}} \in k$ e $k\left(\overline{\mathbf{s}}, \overline{\phi_{X}}\right)=$ $k\left(\overline{\phi_{X}}\right)$. O teste de igualdade de corpos acima mencionado pode ser simplificado ainda mais se exigirmos que os graus das extensões $k(\alpha)$ sobre $k$ sejam todos primos, pois neste caso não haveria corpos intermediários entre $k(\alpha) \mathrm{e}$ $k\left(\overline{\phi_{X}}\right)$, logo estes dois corpos seriam iguais se, e somente se, $\overline{\phi_{X}} \notin k$.

Um roteiro alternativo seria utilizar este método para atacar o problema modular. Neste caso, devemos substituir (i) e (ii) por

(iv) para cada especialização $\mathrm{x} \leftarrow \alpha$ da família, o problema de decidir se $\left|\operatorname{Gal}\left(k(\alpha) / k\left(\overline{\mathbf{s}}, \overline{\phi_{X}}\right)\right)\right|$ é divisível por um dado inteiro $p>1$ está em NP;

(v) se $p$ divide $\left|\operatorname{Gal}\left(L / k\left(\mathrm{~s}, \phi_{X}\right)\right)\right|$, então existe uma especialização da família para a qual o grupo de Galois associado é divisível por $p$ também.

A exigência (v) é um tanto extremada, sendo mais razoável se nos restringirmos a valores primos de $p$. Observe que, neste caso, as simplificações sugeridas no parágrafo anterior surgem naturalmente: para detectar grupos com ordem $p, p$ primo, utilizamos extensões de grau mesmo $p$.

\subsection{Um exemplo}

Dizem que um exemplo fala mais do que mil palavras, então deixem-nos tentar desta forma. Vamos ilustrar uma aplicação do teorema anterior com o seguinte grafo $X$ :

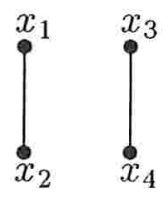


O polinômio associando a este grafo é $\phi\left(x_{1}, x_{2}, x_{3}, x_{4}\right)=x_{1} x_{2}+x_{3} x_{4}$. Seja $\zeta$ uma raiz quinta primitiva da unidade. Utilizando a especialização $x_{i}=\zeta^{i}$, $i=1,2,3,4$, temos que $\bar{\phi}=\zeta^{2}+\zeta^{3}$. Observe que $\overline{s_{i}} \in \mathbb{Q}$, pois $\zeta^{i}$ são raízes do polinômio $x^{4}+x^{3}+x^{2}+x+1 \in \mathbb{Q}[x]$. Como $\mathbb{Q}\left(\zeta, \zeta^{2}, \zeta^{3}, \zeta^{4}\right)=\mathbb{Q}(\zeta)$, temos que

$$
\operatorname{Gal}(\mathbb{Q}(\zeta) / \mathbb{Q}(\bar{\phi}))=\operatorname{Gal}\left(\mathbb{Q}(\zeta) / \mathbb{Q}\left(\zeta^{2}+\zeta^{3}\right)\right)=\{1, \sigma\},
$$

em que $\sigma(\zeta)=\zeta^{4}$, é um subgrupo de $\operatorname{Aut}(X)$.

Assim, o fato de que $\operatorname{Gal}\left(\mathbb{Q}(\zeta) / \mathbb{Q}\left(\zeta^{2}+\zeta^{3}\right)\right)$ não é trivial é um certificado de que $\operatorname{Aut}(X)$ também não é. Naturalmente, podemos exibir um automorfismo explícito como prova de não trivialidade, porém o método permite encontrar tal automorfismo indiretamente, desde que se disponha de algoritmos eficientes para encontrar automorfismos da extensão de corpos obtida após a especialização. Observe que a ação de $\sigma$ sobre $\left\{\zeta, \zeta^{2}, \zeta^{3}, \zeta^{4}\right\}$ é $\left(\zeta, \zeta^{2}, \zeta^{3}, \zeta^{4}\right)^{\sigma}=$ $\left(\zeta^{4}, \zeta^{3}, \zeta^{2}, \zeta\right)$, que corresponde à permutação $(14)(23) \in \operatorname{Aut}(X)$.

Note que uma outra rotulação

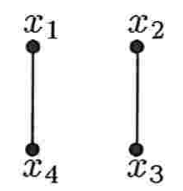

dá origem a um outro polinômio $\phi\left(x_{1}, x_{2}, x_{3}, x_{4}\right)=x_{1} x_{4}+x_{2} x_{3}$. A mesma especialização acima resulta em $\bar{\phi}=2, \operatorname{logo}$

$$
\operatorname{Gal}(\mathbb{Q}(\zeta) / \mathbb{Q}(\bar{\phi}))=\operatorname{Gal}(\mathbb{Q}(\zeta) / \mathbb{Q})=\left\{1, \tau, \tau^{2}, \tau^{3}\right\},
$$

em que $\tau(\zeta)=\zeta^{2}$. Neste caso, obtemos um subgrupo $H$ maior de $\operatorname{Aut}(X)$. O grupo $\operatorname{Aut}(X)$ tem, na verdade, 8 automorfismos.

\subsection{Demonstrando o teorema principal}

Vamos agora demonstrar o teorema que motivou esta abordagem. Inicialmente, observemos que a extensão $L / K\left(\phi_{X}\right)$ é separável, logo, pelo teorema do elemento primitivo, existe $\theta \in L$ tal que $L=K\left(\phi_{X}, \theta\right)$. Podemos tomar $\theta$ como sendo qualquer elemento que não é fixo por nenhuma permutação de $\mathfrak{S}_{n}$. Por exemplo, se char $k=0, \theta=x_{1}+2 x_{2}+\cdots+n x_{n}$ dará conta do recado. No que segue, para simplificar alguns cálculos, vamos supor que $\theta \in k[\mathrm{x}]$. 
Seja $G=\operatorname{Gal}\left(L / K\left(\phi_{X}\right)\right)$. Podemos escrever os conjugados $\sigma(\theta)$ de $\theta$, $\sigma \in G$, como $\sigma(\theta)=h_{\sigma}(\theta)$, para algum polinômio $h_{\sigma}(x) \in K\left(\phi_{X}\right)[x]$. Seja $f_{\theta}(x) \in K\left(\phi_{X}\right)[x]$ o polinômio minimal de $\theta$. Temos

$$
f_{\theta}(x)=\prod_{\sigma \in G}(x-\sigma(\theta))=\prod_{\sigma \in G}\left(x-h_{\sigma}(\theta)\right)
$$

Agora seja $P$ um ideal primo de $k[\mathrm{x}]$, satisfazendo algumas condições que serão explicitadas conforme for preciso. Nossa primeira exigência é

(i) $P$ não divide nenhum dos denominadores dos coeficientes de $f_{\theta}(x)$ e de $h_{\sigma}(x)$.

Seja $k[\mathrm{x}]_{P}$ a localização de $k[\mathrm{x}]$ em $P$ e $\mathcal{M}_{P}=P k[\mathrm{x}]_{P}$ seu ideal maximal. Denotando por $\bar{x}$ a imagem de $x$ pelo homomorfismo canônico de $k[\mathrm{x}]_{P}$ no corpo $E=k[\mathrm{x}]_{P} / \mathcal{M}_{P}$, podemos escrever

$$
\begin{aligned}
\overline{f_{\theta}}(x) & =\prod_{\sigma \in G}(x-\overline{\sigma(\theta)})=\prod_{\sigma \in G}\left(x-\overline{h_{\sigma}(\theta)}\right) \\
& =\prod_{\sigma \in G}\left(x-\overline{h_{\sigma}}(\bar{\theta})\right)
\end{aligned}
$$

Observe que os coeficientes de $\overline{f_{\theta}}(x)$ estão no corpo de frações de $k\left[\overline{\mathbf{s}}, \overline{\phi_{X}}\right]$, que doravante representaremos por $F$. Isto mostra que $\bar{\theta} \in E$ é algébrico sobre $F$. Gostaríamos que $E=F(\bar{\theta})$, o que pode ser conseguido exigindo-se que

(ii) $P$ não contém nenhum dos denominadores de $g_{i}(x)$, em que $g_{i}(x) \in$ $K\left(\phi_{X}\right)[x]$ são tais que $x_{i}=g_{i}(\theta)$.

Assim, temos que $\overline{x_{i}}=\overline{g_{i}}(\bar{\theta})$ e como $\overline{x_{i}}$ geram $E$ sobre $k$ e, a fortiori, $E$ sobre $F$, temos que $E=F(\bar{\theta})$. Logo $E / F$ é uma extensão finita, ainda mais, é galoisiana, já que os conjugados de $\bar{\theta}$ estão entre as raízes de $\overline{f_{\theta}}(x)$, isto é, entre $\overline{h_{\sigma}}(\bar{\theta}) \in E$.

Agora seja

$$
H=\left\{\sigma \in G \mid \overline{h_{\sigma}}(\bar{\theta}) \text { é conjugado de } \bar{\theta} \text { sobre } F\right\} .
$$

Vamos mostrar que $H$ é um subgrupo de $G$ que é isomorfo a $\operatorname{Gal}(E / F)$. Mas não sem antes aumentarmos nossa lista de exigências: 
(iii) $\mathcal{M}_{P}$ não contém $\Delta\left(f_{\theta}\right)$, o discriminante de $f_{\theta}$.

Vamos descrever este isomorfismo. A cada $\sigma \in \operatorname{Gal}(E / F)$, podemos associar um $\imath(\sigma) \in H$ tal que

$$
\sigma(\bar{\theta})=\overline{h_{\imath(\sigma)}}(\bar{\theta})
$$

Devemos mostrar primeiramente que esta aplicação está bem definida, ou seja, que este $\imath(\sigma)$ é único. Suponha que não. Temos

$$
\overline{h_{\tau}}(\bar{\theta})=\overline{h_{\tau^{\prime}}}(\bar{\theta}) \Longleftrightarrow \overline{h_{\tau}(\theta)}=\overline{h_{\tau^{\prime}}(\theta)} \Longleftrightarrow h_{\tau}(\theta)-h_{\tau^{\prime}}(\theta) \in \mathcal{M}_{P}
$$

Mas neste caso, como $h_{\tau}(\theta)-h_{\tau^{\prime}}(\theta)$ divide $\Delta\left(f_{\theta}\right)$, teríamos $\Delta\left(f_{\theta}\right) \in \mathcal{M}_{P}$, o que contraria nossa exigência (iii).

A demonstração acima mostra ainda que a aplicação $\imath: \operatorname{Gal}(E / F) \rightarrow H$ é injetora. Por outro lado, $\imath$ é claramente sobrejetora, pois, para $\sigma \in H$, $\bar{\theta} \mapsto \overline{h_{\sigma}}(\bar{\theta})$ define um elemento de $\operatorname{Gal}(E / F)$ com imagem $\sigma$.

Falta mostrar que $\imath$ preserva a operação do grupo. Precisaremos do seguinte resultado.

\section{Teorema 4.2 Temos}

$$
h_{\tau \sigma}(\theta)=h_{\sigma} \circ h_{\tau}(\theta)
$$

para quaisquer $\sigma, \tau \in G$.

\section{Demonstração Temos}

$$
\begin{aligned}
h_{\tau \sigma}(\theta) & =\tau \sigma(\theta)=\tau\left(h_{\sigma}(\theta)\right) \\
& =h_{\sigma}(\tau(\theta))=h_{\sigma}\left(h_{\tau}(\theta)\right)
\end{aligned}
$$

Para $\sigma, \tau \in \operatorname{Gal}(E / F)$, temos

$$
\begin{aligned}
\overline{h_{\imath(\sigma \tau)}}(\bar{\theta}) & =\sigma \tau(\bar{\theta})=\sigma\left(\overline{h_{\imath(\tau)}}(\bar{\theta})\right) \\
& =\overline{h_{\imath(\tau)}}(\sigma(\bar{\theta}))=\overline{h_{\imath(\tau)}}\left(\overline{h_{\imath(\sigma)}}(\bar{\theta})\right) \\
& =\overline{h_{\imath(\tau)} \circ h_{\imath(\sigma)}(\theta)}=\overline{h_{\imath(\sigma)_{\imath(\tau)}}(\theta)} \\
& =\overline{h_{\imath(\sigma) \imath(\tau)}}(\bar{\theta})
\end{aligned}
$$

Pela unicidade provada acima, temos $\imath(\sigma \tau)=\imath(\sigma) \imath(\tau)$. Logo concluímos que $\imath$ é um isomorfismo e, conseqüentemente, podemos considerar $\operatorname{Gal}(E / F) \cong H$ como um subgrupo de $G$, como afirmamos acima.

Resumindo, demonstramos o seguinte resultado: 
Teorema 4.3 Existe um polinômio $m(\mathrm{x}) \in k[\mathrm{x}]$ tal que, para qualquer ideal primo $P$ de $k[\mathrm{x}]$ que não divide $m_{X}(\mathrm{x})$, o grupo de Galois $\operatorname{Gal}(E / F)$ é um subgrupo de $\operatorname{Gal}\left(L / K\left(\phi_{X}\right)\right)$.

De fato, o polinômio $m(\mathrm{x})$ nada mais é do que o produto dos diversos polinômios da lista de exigências acima.

Finalmente, para obter o resultado procurado sobre especializações, basta uma escolha adequada de $P$. Por exemplo, se $\alpha \in k$, a especialização $\mathrm{x} \leftarrow \alpha$ corresponde a tomar $P=\left(x_{1}-\alpha_{1}, \ldots, x_{n}-\alpha_{n}\right)$. Se $\alpha$ está em uma extensão algébrica finita de $k$, digamos $k^{\prime}$, considere o homomorfismo $\varphi: k[\mathrm{x}] \rightarrow k^{\prime}$ tal que $\varphi\left(x_{i}\right)=\alpha_{i}$. A especialização $\mathrm{x} \leftarrow \alpha$ pode ser obtida tomando-se $P=\operatorname{ker} \varphi$.

O motivo para trabalharmos com este teorema mais geral é ter em mãos um resultado que possa ser aplicado no maior número de situações possíveis, uma vez que ainda não se conhece uma família de especializações com as propriedades desejadas. O teorema acima diz que a maioria destas especializações funciona, a menos de um conjunto $Z$ "pequeno", dos ideais primos de $k[\mathrm{x}]$ que não dividem um polinômio $m(\mathrm{x})$. Na linguagem da Álgebra Comutativa, $Z=V(m)$ é um fechado na topologia de Zariski de $\operatorname{Spec}(k[\mathrm{x}])$.

\subsection{Obtendo explicitamente $m(\mathrm{x})$}

Podemos obter uma descrição mais explícita do polinômio $m(\mathrm{x})$ do teorema principal. Para isto, é necessário estudar os possíveis denominadores de um elemento de $L$ quando escrito como uma expressão em $\theta$ com coeficientes em $K(\phi)$. É o que faremos em seguida.

Observe inicialmente que todos os elementos de $L$ dos quais fazemos uso na demonstração do teorema principal são inteiros sobre $k[\mathbf{s}]$. Assim, parece razoável esperar que se $p(\theta) \in L, p(x) \in K(\phi)[x]$, então todos os coeficientes de $p(x)$ estejam em $k[\mathbf{s}, \phi]$. Entretanto, isto é falso. Embora $K(\phi)[x]=$ $K[\phi][x]$, pois $\phi$ é inteiro sobre $k[\mathbf{s}]$, nem sempre é possível nos livrarmos dos polinômios simétricos dos denominadores.

Por exemplo, para $\phi=x_{1} x_{2}+x_{2} x_{3} \in L=\mathbb{Q}\left[x_{1}, x_{2}, x_{3}\right]$, temos $\operatorname{Gal}(L / K(\phi))=$ $\{1,(13)\}$; e como $x_{2}$ é fixado por todas as permutações do grupo de Galois, sabemos que $x_{2} \in K(\phi)$. De fato,

$$
x_{2}=\frac{x_{1} x_{2} x_{3}}{x_{1} x_{3}}=\frac{s_{3}}{s_{2}-\phi}
$$


Os conjugados de $\phi$ são $x_{1} x_{3}+x_{2} x_{3}, x_{1} x_{3}+x_{1} x_{2}$ e o próprio $\phi$. Então $\phi$ satisfaz

$$
\begin{aligned}
p(t) & =\left(t-x_{1} x_{2}-x_{2} x_{3}\right)\left(t-x_{1} x_{3}-x_{2} x_{3}\right)\left(t-x_{1} x_{3}-x_{1} x_{2}\right) \\
& =t^{3}-2 s_{2} \cdot t^{2}+\left(s_{2}^{2}+s_{1} s_{3}\right) \cdot t-\left(s_{1} s_{2} s_{3}-s_{3}^{2}\right)
\end{aligned}
$$

Utilizando o algoritmo de Euclides, podemos escrever $a(t) \cdot\left(s_{2}-t\right)+b(t)$. $p(t)=1$ para remover $\phi$ do denominador. O resultado final é

$$
x_{2}=s_{3} \cdot a(\phi)=\frac{\phi^{2}-s_{2} \cdot \phi+s_{1} s_{3}}{s_{3}}
$$

Assim, $x_{2}$ está, na verdade, em $k\left[\mathrm{~s}, \phi, 1 / s_{3}\right]$.

Embora nem sempre obtenhamos coeficientes em $k[\mathbf{s}, \phi]$, a situação não é tão ruim assim:

Teorema 4.4 Seja $\theta \in k[\mathrm{x}]$ tal que $L=K(\theta)$.

(a) Existe um polinômio $m(\mathrm{x}) \in k[\mathrm{x}]$ tal que, se $\alpha \in k[\mathrm{x}]$, então $\alpha=h(\theta)$ para algum polinômio $h(x)$ com coeficientes em $k[\mathrm{~s}, 1 / \mathrm{m}]$. Podemos ainda tomar

$$
q(\mathbf{x})=\prod_{\substack{\sigma, \tau \in \mathfrak{G}_{n} \\ \sigma \neq \tau}}(\sigma(\theta)-\tau(\theta))
$$

(b) Existe um polinômio $r(\mathrm{x}) \in k[\mathrm{x}]$ tal que, se $\alpha \in k[\mathrm{x}]$, então $\alpha=h(\theta)$ para algum polinômio $h(x)$ com coeficientes em $k[\mathbf{s}, \phi, 1 / r]$. Podemos ainda tomar

$$
r(\mathrm{x})=q(\mathrm{x}) \cdot \prod_{\substack{\sigma, \tau \in C \\ \sigma \neq \tau}}(\sigma(\phi)-\tau(\phi))
$$

em que $C$ é um conjunto de representantes de classes laterais de $\mathfrak{S}_{n}$ módulo $\operatorname{Gal}(L / K(\phi))$.

\section{Demonstração}

(a) Seja

$$
\alpha=a_{0}+a_{1} \theta+a_{2} \theta^{2}+\cdots+a_{d-1} \theta^{d-1}, \quad a_{i} \in K,
$$

em que $d=[L: K]$. Aplicando $\sigma_{j} \in G=\operatorname{Gal}(L / K)$, temos

$$
\sigma_{j}(\alpha)=a_{0}+a_{1} \sigma_{j}(\theta)+a_{2} \sigma_{j}(\theta)^{2}+\cdots+a_{d-1} \sigma_{j}(\theta)^{d-1}, \quad \sigma_{j} \in G
$$


Mas como \# $G=d$, temos um sistema de $d$ incógnitas $a_{i}$ e $d$ equações. Como $\alpha \in k[\mathrm{x}], \sigma_{j}(\alpha) \in k[\mathrm{x}]$ para todo $\sigma_{j} \in G$. Assim, os denominadores de $a_{i}$ dividem o determinante de Vandermonde

$$
\operatorname{det}\left(\sigma_{j}(\theta)^{i}\right)=\prod_{1 \leq i<j \leq d}\left(\sigma_{i}(\theta)-\sigma_{j}(\theta)\right)
$$

Porém este determinante não está necessariamente em $k[\mathrm{~s}]$. Mas é fácil consertar: basta tomar

$$
m(\mathrm{x})=\prod_{\substack{\sigma, \tau \in \sigma_{n} \\ \sigma \neq \tau}}(\sigma(\theta)-\tau(\theta))
$$

Como $\theta \in k[\mathrm{x}]$, temos que $m(x)$ também é um polinômio em $k[\mathrm{x}]$. Assim, podemos escrever cada $a_{i}$ como um quociente $p(\mathrm{x}) / m(\mathrm{x})$ para algum polinômio $p(\mathrm{x})$. Além disso, como $m(\mathrm{x})$ e $a_{i}$ são simétricos em $x_{1}, \ldots, x_{n}$, temos que $p(\mathrm{x})$ também é. Logo $m(\mathrm{x})$ e $p(\mathrm{x})$ são ambos polinômios simétricos e, portanto, estão em $k[\mathrm{~s}]$, o que encerra a demonstração neste caso.

(b) Este caso é análogo ao do item (a). Escrevemos

$$
\alpha=a_{0}+a_{1} \theta+a_{2} \theta^{2}+\cdots+a_{d-1} \theta^{d-1}, \quad a_{i} \in K(\phi)=K[\phi],
$$

em que $d=[L: K(\phi)]$. Aplicando o raciocínio anterior, temos que cada $a_{i}$ é o quociente de dois polinômios $p(\mathrm{x})$ e $m(\mathrm{x})$ invariantes por $\operatorname{Gal}(L / K(\phi))$ (podemos até tomar o mesmo $m(\mathrm{x})$ do item anterior, que é o que faremos).

Agora, repetimos o processo com $\phi$ no lugar de $\theta$. Sendo $e$ o grau do polinômio minimal de $\phi$ sobre $K$, escrevemos

$$
m(\mathrm{x}) \cdot a_{i}=b_{0}+b_{1} \phi+\cdots+b_{e-1} \phi^{e-1}, \quad b_{j} \in K
$$

Sabemos que $m(\mathrm{x}) \cdot a_{i} \in k[\mathrm{x}]$, então podemos tentar aplicar novamente o método acima. Porém, desta vez, não podemos utilizar os automorfismos de $K(\phi) / K$, uma vez que esta extensão não é, necessariamente, galoisiana. Em vez disto, escolhemos representantes $\sigma_{1}, \ldots, \sigma_{e}$ de $\mathfrak{S}_{n} / \operatorname{Gal}(L / K(\phi))$, isto é, permutações tais que $\sigma_{j}(\phi)$ sejam todos os conjugados de $\phi$. O resto da demonstração segue, mutatis mutandis, a anterior. 
Utilizando este resultado, podemos computar explicitamente o polinômio $m(\mathrm{x})$ do teorema principal. Infelizmente este cálculo envolve os conjugados de $\phi$, que só sabemos calcular conhecendo-se um conjunto de representantes de classes laterais $C$ como acima.

\subsection{Escolhendo especializações}

Embora não se saiba como construir a família de especializações com as propriedades requeridas para resolver o problema de certificar a trivialidade do grupo de automorfismos de um grafo, vamos considerar nesta seção uma tentativa de especialização, examinando as dificuldades e os problemas que surgem em sua construção.

Vamos considerar especializações em corpos finitos. Tomamos $k=\mathbb{Z} / p \mathbb{Z}$ para algum primo $p$ e faremos especializações $\mathrm{x} \leftarrow \alpha$ com $\alpha_{i}$ em alguma extensão finita de $k$. Sob o ponto de vista computacional, esta escolha justificase pela facilidade em representar e operar eficientemente corpos finitos em um computador.

Se $\mathbb{F}_{q}$ denota o corpo com $q=p^{d}$ elementos, então Gal $\left(\mathbb{F}_{q} / k\right)$ é simplesmente o grupo cíclico de ordem $d$, gerado pelo automorfismo de Frobenius : $\varphi(x)=x^{p}$. Desta forma, este grupo apresenta uma estrutura bastante simples, o que facilitará nossa análise.

Outra vantagem de se trabalhar com especializações desta forma é que, para certificar a trivialidade do grupo de automorfismos de um grafo $X$, basta mostrar que \# Aut $(X)$ não é divisível por nenhum primo $d$ menor ou igual a $n=\# V(X)$. Por outro lado, se $d \mid \# \operatorname{Aut}(X)$, então, pelo teorema de Sylow, existe um subgrupo de \# Aut $(X)$ de ordem $d$. O que tentaremos, portanto, é obter uma quantidade suficientemente grande de especializações de modo que se $\operatorname{Aut}(X)$ possuir um subgrupo $H$ de ordem $d, d$ primo, então $H$ corresponde ao subgrupo obtido por uma especialização por elementos em $\mathbb{F}_{q}$ (note que $\operatorname{Gal}\left(\mathbb{F}_{q} / k\right)$ também é cíclico de ordem $d$ ).

Tais especializações de fato existem. Suponha que $\operatorname{Aut}(X)$ possua um elemento $\sigma$ de ordem $d, d$ primo. Então $\sigma$ é o produto de ciclos de tamanho $d$, digamos

$\sigma=(1,2, \ldots, d)(d+1, d+2, \ldots 2 d-1) \ldots((r-1) d+1,(r-1) d+2, \ldots r d-1)$

Seja agora $p(x)$ um polinômio de grau $d$ irredutível em $k[x]$ e seja $\alpha$ uma. 
de suas raízes. Fazemos a especialização

$$
\begin{array}{ll}
x_{i} \leftarrow \alpha^{p^{i-1}} & \text { para } 1 \leq i<r d \\
x_{i} \leftarrow 1 & \text { para } i \geq r d
\end{array}
$$

Como esta especialização corresponde às raízes de $(x-1)^{n-r d}(p(x))^{r}$, temos que os polinômios simétricos especializados $\overline{s_{i}}$ serão os coeficientes deste polinômio, que pertencem a $k$. Por outro lado, como $\sigma\left(\phi_{X}\right)=\phi_{X}$, temos que $\overline{\sigma\left(\phi_{X}\right)}=\overline{\phi_{X}}$. Porém, temos que $\overline{\sigma\left(\phi_{X}\right)}$ é nada mais nada menos do que $\left(\overline{\phi_{X}}\right)^{p}$. Em outras palavras, $\overline{\phi_{X}}$ é fixo pelo automorfismo de Frobenius $\varphi$ e, portanto, pertence a $k$ também. Assim, o subgrupo de $\operatorname{Aut}(X)$ obtido por esta especialização é $\operatorname{Gal}(k(\alpha), k)=\operatorname{Gal}\left(F_{q} / k\right)=\langle\varphi\rangle$.

A especialização acima foi, entretanto, obtida a partir de uma particular rotulação dos vértices e do conhecimento da ação de $\sigma$ sobre estes. Não podemos simplesmente testar todos os elementos de ordem $d$ : há $n(n-$ 1) $\ldots(n-d+1) / d$ ciclos de tamanho $d$, o que já é muito, uma vez que devemos testá-los para todos os primos $d$ menores ou iguais a $n$.

Por outro lado, como $\left[F_{q}: k\right]=d$ é primo, temos que $k(\alpha)=k\left(\overline{\mathbf{s}}, \overline{\phi_{x}}\right)$ ou $k=k\left(\overline{\mathrm{s}}, \overline{\phi_{x}}\right)$. Assim, o teste se resume a verificar se $\overline{\mathrm{s}}$ e $\overline{\phi_{x}}$ estão em $k$ ou não. Resta a dúvida de se é ou não possível obter um número polinomial de tais especializações sem conhecimento prévio de $\operatorname{Aut}(X)$ de modo que elas possam, de fato, certificar a trivialidade deste grupo. 


\section{Apêndice A}

\section{Notações}

\section{A.1 Geral}

- $|X|, \# X$ : cardinalidade do conjunto $X$

\section{A.2 Grafos}

Nesta seção $X$ denota um grafo. Se $v$ e $w$ são vértices adjacentes de $X$, utilizaremos a notação $v w$ para indicar a aresta que liga estes dois vértices.

- $V(X)$ : conjunto dos vértices de $X$

- $E(X)$ : conjunto das arestas de $X$

- Aut $(X)$ : grupo de automorfismos de $X$

- $(X, \lambda)$ : grafo $X$ rotulado pela função $\lambda: V(X) \rightarrow L$, em que $L$ é um conjunto de rótulos

\section{A.3 Grupos}

Nesta seção $G, H, N$ denotam grupos.

- $[G: H]$ : índice de $H$ em $G$

- $H \triangleleft G$ : $H$ é um subgrupo normal de $G$

- $\mathbf{Z}_{G}(N)$ : centralizador de $N$ em $G$ 


\section{A.4 Corpos e polinômios}

- $k[\mathrm{x}]$ : anel dos polinômios nas variáveis $x_{1}, x_{2}, \ldots, x_{n}$

- $k(\mathrm{x})$ : corpo das funções racionais nas variáveis $x_{1}, x_{2}, \ldots, x_{n}$

- $\mathrm{s}$ : polinômios simétricos fundamentais $s_{1}=x_{1}+x_{2}+\cdots+x_{n}, s_{2}=$ $x_{1} x_{2}+x_{1} x_{3}+\cdots+x_{n-1} x_{n}, \ldots, s_{n}=x_{1} x_{2} \ldots x_{n}$ nas variáveis $x_{1}, x_{2}, \ldots, x_{n}$

- $p(\mathrm{x})$ : polinômio $p\left(x_{1}, x_{2}, \ldots, x_{n}\right)$

- $E / F$ : extensão de corpos $E$ sobre $F$

- $\operatorname{Gal}(E / F)$ : grupo de Galois de $E / F$

\section{A.5 Anéis e localização}

- $R_{P}$ : localização do anel $R$ no ideal primo $P$

- $\mathcal{M}_{P}$ : ideal maximal do anel local obtido a partir da localização em $P$

Um ideal $P$ é primo se $x y \in P$ implica $x \in P$ ou $y \in P$. Um anel local é um anel com um único ideal maximal. A localização $R_{P}$ de $R$ em $P$ é o anel de frações $x / s, \operatorname{com} x \in R$ e $s \notin P$, para os quais vale

$$
\frac{x}{s}=\frac{y}{s^{\prime}} \Longleftrightarrow \exists t \notin P \mid t\left(x s^{\prime}-y s\right)=0
$$

No caso de domínios, podemos utilizar a condição mais simples $x s^{\prime}=y s$. A localização, como o próprio nome diz, produz anéis locais, focalizando sobre um único primo e transformando o resto em unidades do anel. 


\section{Apêndice B}

\section{Teoria de Galois}

Apresentamos aqui alguns resultados básicos da teoria de Galois que são utilizados neste trabalho. Os resultados são clássicos mas a abordagem difere substancialmente de textos como [Jac], de modo que incluímos diversas provas. Para simplificar a exposição, enunciaremos os resultados para $\mathbb{Q}$, porém é fácil ver que os resultados se estendem ipsis literis para corpos de característica 0 e mesmo para corpos de característica positiva.

Para maiores detalhes e outras abordagens, consulte qualquer livro de Álgebra, como por exemplo [Jac].

\section{B.1 Polinômios simétricos}

O primeiro conceito de que necessitaremos é o de polinômio simétrico : um polinômio é simétrico se ele não se altera quando intercambiamos duas de suas variáveis. Provavelmente os polinômios simétricos mais simples são os chamados de polinômios simétricos elementares :

$$
\begin{aligned}
s_{1} & =x_{1}+x_{2}+\cdots+x_{n} \\
s_{2} & =x_{1} x_{2}+x_{1} x_{3}+\cdots+x_{n-1} x_{n} \\
s_{3} & =x_{1} x_{2} x_{3}+x_{1} x_{2} x_{4}+\cdots+x_{n-2} x_{n-1} x_{n} \\
& \vdots \\
s_{n} & =x_{1} \ldots x_{n-1} x_{n}
\end{aligned}
$$

Utilizando os polinômios simétricos elementares, é fácil produzir outros polinômios simétricos, tais como $s_{2}+s_{3}, s_{1}^{2} s_{n}$ e, em geral, qualquer polinômio em 
$s_{1}, s_{2}, \ldots, s_{n}$. O fato interessante é que esta é a única maneira de produzir polinômios simétricos. A demonstração é simples e a reproduzimos aqui.

Em primeiro lugar, ordenamos os monômios segundo uma ordem graulexicográfica, isto é, diremos que $x_{1}^{\alpha_{1}} x_{2}^{\alpha_{2}} \ldots x_{n}^{\alpha_{n}}>x_{1}^{\beta_{1}} x_{2}^{\beta_{2}} \ldots x_{n}^{\beta_{n}}$ se o grau $\sum \alpha_{i}$ do primeiro monômio for maior do que o grau $\sum \beta_{i}$ do segundo ou, se os graus forem iguais, o primeiro for lexicograficamente maior do que o segundo (em outras palavras, existe um $k$ tal que $\alpha_{i}=\beta_{i}$ para $1 \leq i<k$ e $\alpha_{k}>\beta_{k}$ ). O termo líder de um polinômio é o maior de seus monômios juntamente com seu coeficiente. Por exemplo, o termo líder de

$f\left(x_{1}, x_{2}, x_{3}\right)=2 x_{1}^{2} x_{2}+2 x_{1}^{2} x_{3}+2 x_{2}^{2} x_{1}+2 x_{2}^{2} x_{3}+2 x_{3}^{2} x_{1}+2 x_{3}^{2} x_{2}+x_{1}+x_{2}+x_{3}$ é $2 x_{1}^{2} x_{2}$.

Agora, dado um polinômio simétrico $p\left(x_{1}, \ldots, x_{n}\right)$, seja $c x_{1}^{\alpha_{1}} x_{2}^{\alpha_{2}} \ldots x_{n}^{\alpha_{n}}$ o seu termo líder. Já que $p$ é simétrico, devemos ter $\alpha_{1} \geq \alpha_{2} \geq \cdots \geq \alpha_{n}$. Utilizando os polinômios simétricos elementares, podemos construir outro polinômio simétrico com mesmo termo líder: basta tomar

$$
c \cdot s_{1}^{\alpha_{1}-\alpha_{2}} s_{2}^{\alpha_{2}-\alpha_{3}} \ldots s_{n-1}^{\alpha_{n-1}-\alpha_{n}} s_{n}^{\alpha_{n}}
$$

Agora $p-c s_{1}^{\alpha_{1}-\alpha_{2}} s_{2}^{\alpha_{2}-\alpha_{3}} \ldots s_{n-1}^{\alpha_{n-1}-\alpha_{n}} s_{n}^{\alpha_{n}}$ é um polinômio simétrico com termo líder menor. Repetindo o processo quantas vezes for necessário, obteremos eventualmente 0 , ou seja, desta forma teremos escrito $p$ como polinômio em $s_{1}, s_{2}, \ldots, s_{n}$. No exemplo acima, temos

$$
f\left(x_{1}, x_{2}, x_{3}\right)-2 s_{1} s_{2}=-3 x_{1} x_{2} x_{3}+x_{1}+x_{2}+x_{3}
$$

com termo líder $-3 x_{1} x_{2} x_{3}<2 x_{1}^{2} x_{2}$. Continuando o processo, obtemos finalmente

$$
f\left(x_{1}, x_{2}, x_{3}\right)=2 s_{1} s_{2}-3 s_{3}+s_{1}
$$

A partir da expressão

$$
\prod_{1 \leq i \leq n}\left(x-x_{i}\right)=x^{n}-s_{1} x^{n-1}+s_{2} x^{n-2}-\cdots+(-1)^{n} s_{n}
$$

temos um importante corolário do resultado acima: qualquer expressão simétrica das raizes de um polinômio com coeficientes em um corpo $k$ pertence também $a k$.

Lembramos que um elemento $\alpha$ é algébrico sobre um corpo $L$ se $\alpha$ é raiz de um polinômio com coeficientes em $L$. Como um exemplo, vamos aplicar 
o corolário para provar que se $\alpha$ é algébrico sobre um corpo $L$ que, por sua vez, é algébrico sobre $\mathbb{Q}$, então $\alpha$ é algébrico sobre $\mathbb{Q}$ (isto é mais difícil de enunciar do que provar!)

Em primeiro lugar, $\alpha$ é raiz de um polinômio

$$
\alpha^{n}+l_{n-1} \cdot \alpha^{n-1}+l_{n-2} \cdot \alpha^{n-2}+\cdots+l_{0}=0
$$

$\operatorname{com} l_{i} \in L$. Cada $l_{i}$ é algébrico sobre $\mathbb{Q}$, então é raiz de um polinômio $p_{i}(x)$ com coeficientes em $\mathbb{Q}$. Agora considere o produto

$$
P(x)=\prod_{l_{n-1}^{\prime}, l_{n-2}^{\prime}, \ldots, l_{0}^{\prime}}\left(x^{n}+l_{n-1}^{\prime} \cdot x^{n-1}+l_{n-2}^{\prime} \cdot x^{n-2}+\cdots+l_{0}^{\prime}\right)
$$

em que cada $l_{i}^{\prime}$ assume todas as raízes de $p_{i}(x)$. Obviamente os coeficientes do polinômio $P(x)$ são expressões simétricas das raízes de $p_{i}(x)$ e portanto pertencem a $\mathbb{Q}$. Como $P(\alpha)=0$ concluímos que $\alpha$ é de fato algébrico sobre $\mathbb{Q}$.

\section{B.2 Imersões}

$\mathrm{Se}$

$$
\left(\frac{1}{2-3 i}+5 i+\frac{1}{7}\right) \cdot \frac{(2+i) \cdot\left(\frac{3}{4}-5 i\right)}{7+13 i}=\frac{276099-158443 i}{79352}
$$

você pode dizer o valor de

$$
\left(\frac{1}{2+3 i}-5 i+\frac{1}{7}\right) \cdot \frac{(2-i) \cdot\left(\frac{3}{4}+5 i\right)}{7-13 i} ?
$$

É fácil! Como a segunda expressão é conjugada da primeira, não precisamos repetir as contas novamente, a resposta é $(276099+158443 i) / 79352$ simplesmente. Este fenômeno merece uma atenção maior.

As propriedades da conjugação $\tau(a+b i)=a-b i, a, b \in Q$, que utilizamos acima são $\tau\left(z_{1}+z_{2}\right)=\tau\left(z_{1}\right)+\tau\left(z_{2}\right), \tau\left(z_{1} z_{2}\right)=\tau\left(z_{1}\right) \tau\left(z_{2}\right)$ e $\tau(z)=z$ para $z \in$ $Q$. Dizemos que uma função $\tau: L \rightarrow \overline{\mathbb{Q}}$ satisfazendo estas propriedades é uma imersão do corpo $L$ em $\overline{\mathbb{Q}}$ sobre $Q$. A função $\tau$ leva $i$ em $-i$, que são as raízes de $x^{2}+1$, um polinômio irredutível com coeficientes em $\mathbb{Q}$. De fato, toda imersão $\sigma$ leva $i$ em $i$ ou $-i$ : basta observar que $i^{2}+1=0 \Rightarrow \sigma(i)^{2}+1=0$, de modo que $\sigma(i)= \pm i$. É claro que não precisamos nos restringir a este caso 
especial; o mesmo ocorre com as raízes de qualquer polinômio irredutível, isto é, qualquer imersão leva uma raiz de um polinômio irredutível em $\mathbb{Q}$ em uma raiz do mesmo polinômio. Em analogia ao caso acima, diremos que raízes de um mesmo polinômio irredutível em $\mathbb{Q}$ são conjugadas. Elas terão um importante papel no que segue.

Agora estamos prontos para caracterizar as imersões de uma extensão algébrica simples, isto é, um corpo $\mathbb{Q}(\alpha)$ gerado sobre $\mathbb{Q}$ por um único elemento algébrico $\alpha$. Uma vez que qualquer elemento de $\mathbb{Q}(\alpha)$ é uma função racional (i.e., o quociente de dois polinômios) com coeficientes em $\mathbb{Q}$, tudo o que precisamos para descrever uma imersão é dizer quem é a imagem de $\alpha$. A discussão acima restringe as possibilidades aos conjugados de $\alpha$. Então por que não tentar definir $\sigma(r(\alpha))=r\left(\alpha^{\prime}\right)$, em que $\alpha^{\prime}$ é qualquer conjugado de $\alpha$ e $r(\alpha)$ é uma função racional em $\alpha$ ? Bem, em primeiro lugar, há várias maneiras de se escrever um elemento como uma função racional (por exemplo $\left.i=i^{2}+i+1=1 /(-i)\right)$, de modo que a função acima pode não estar bem definida. Além disso, ainda precisamos garantir que a função acima é de fato uma imersão. Ambas as questões são tratadas pelo seguinte lema:

Teorema B.1 Se $q(x)$ é um polinômio com coeficientes em $\mathbb{Q}$ tal que $q(\alpha)=$ 0 , então $q\left(\alpha^{\prime}\right)$ para qualquer conjugado $\alpha^{\prime}$ de $\alpha$.

Seja $p(x)$ o polinômio minimal de $\alpha$, isto é, o polinômio de menor grau com coeficientes em $\mathbb{Q}$ do qual $\alpha$ é raiz. Dividindo $q(x)$ por $p(x)$, podemos escrever $q(x)=a(x) \cdot p(x)+r(x)$, em que $r(x)$ é 0 ou tem grau menor do que $p(x)$. Mas já que $q(\alpha)=a(\alpha) \cdot p(\alpha)+r(\alpha) \Rightarrow r(\alpha)=0$, e $p(x)$ é o polinômio minimal de $\alpha$, devemos ter $r(x)=0$, ou seja, $p(x)$ divide $q(x)$. Em particular, se $\alpha$ e $\alpha^{\prime}$ são raízes de um mesmo polinômio irredutível $t(x)$, temos que $t(x)$ é um múltiplo de $p(x)$, ou seja, $t(x)=c \cdot p(x), c \in \mathbb{Q}$. Assim, $\alpha$ e $\alpha^{\prime}$ são raízes de $p(x)$ e, portanto, qualquer raiz de $p(x)$ é também raiz de $q(x)$, o que termina a demonstração.

O resultado acima estende-se de maneira óbvia a funções racionais, de modo que se $r(\alpha)=s(\alpha)$, isto é, $\alpha$ é raiz de $r(x)-s(x)$, então $r\left(\alpha^{\prime}\right)-$ $s\left(\alpha^{\prime}\right)=0$. Desta forma, a função $\sigma$ acima realmente está bem definida. Além disso, se $r(\alpha) s(\alpha)=t(\alpha)$, então $r\left(\alpha^{\prime}\right) s\left(\alpha^{\prime}\right)=t\left(\alpha^{\prime}\right) \Longleftrightarrow \sigma(r(\alpha)) \sigma(s(\alpha))=$ $\sigma(t(\alpha)) \Longleftrightarrow \sigma(r(\alpha)) \sigma(s(\alpha))=\sigma(r(\alpha) s(\alpha))$. Resumimos este importante resultado:

Teorema B.2 Se $\alpha$ é raiz de um polinômio irredutivel $p(x)$ de grau d com 
coeficientes em $\mathbb{Q}$, então existem exatamente d imersões de $\mathbb{Q}(\alpha)$ em $\overline{\mathbb{Q}}$ sobre $\mathbb{Q}$, dadas por $\sigma(\alpha)=\alpha^{\prime}$, em que $\alpha^{\prime}$ é qualquer raiz de $p(x)$.

\section{B.3 Extensões Simples}

Agora que temos uma descrição razoavelmente completa de imersões de extensões simples de $\mathbb{Q}$, o que podemos dizer sobre o resto? Felizmente, podemos reduzir nosso estudo ao caso anterior:

Teorema B.3 (Elemento Primitivo) Seja $L=\mathbb{Q}\left(\gamma_{1}, \gamma_{2}, \ldots, \gamma_{n}\right)$ o corpo gerado por elementos $\gamma_{i}$ algébricos sobre $\mathbb{Q}$. Então existe um elemento $\theta \in L$ tal que $L=\mathbb{Q}(\theta)$.

É suficiente provar o resultado para $n=2$ já que o caso geral segue por indução. Então vamos supor que $L$ é gerado por dois elementos $\alpha$ e $\beta$, que são raízes dos polinômios irredutíveis $p(x)$ e $q(x)$, respectivamente. Denotaremos ainda por $\alpha_{1}=\alpha, \alpha_{2}, \ldots, \alpha_{r}$ e $\beta_{1}=\beta, \beta_{2}, \ldots, \beta_{s}$ os conjugados destes elementos.

Bem, se estamos tentado encontrar um único gerador para $L=\mathbb{Q}(\alpha, \beta)$, por que não tentar inicialmente um elemento da forma $\theta=\alpha+c \beta$ com $c \in Q$ ? Se $\theta$ gera $L$ sobre $\mathbb{Q}$, então existe um polinômio $m(x)$ com coeficientes em $\mathbb{Q}$ tal que $\alpha=m(\theta)$ (e, portanto, $\beta=(m(\theta)-\alpha) / c$ ). Guiados por nossa experiência anterior, sabemos que se $\bar{\theta}$ é conjugado de $\theta$, então $\bar{\alpha}=$ $m(\bar{\theta})$. Mas quem são os conjugados de $\theta$ ? Podemos encontrar facilmente um polinômio que admite $\theta$ como uma de suas raízes. Considere

$$
t(x)=\prod_{i, j}\left(x-\alpha_{i}-c \beta_{j}\right)
$$

Assim, os conjugados de $\theta$ formam um subconjunto de $\left\{\alpha_{i}+c \beta_{j}\right\}$, e seriam exatamente estes elementos se soubéssemos que $t(x)$ é irredutível. Infelizmente, $t(x)$ não precisa ser irredutível. Porém, é ainda possível construir um polinômio $m(x)$ tal que $m\left(\alpha_{i}+c \beta_{j}\right)=\alpha_{i}$. Vejamos como.

Escolhemos $c \in \mathbb{Q}$ tal que $\alpha_{i}+c \beta_{j}$ são todos distintos. Agora, seja $l(\alpha, \beta) \in L$. Considere o seguinte polinômio interpolador de Lagrange

$$
m(x)=\sum_{\sigma \in \mathfrak{S}_{n}} \prod_{\substack{\tau \in \mathfrak{G}_{n} \\ \tau \neq \sigma}} \frac{\left(x-\alpha_{\tau(i)}-c \beta_{\tau(i)}\right)}{\left(\alpha_{\sigma(i)}+c \beta_{\sigma(i)}-\alpha_{\tau(i)}-c \beta_{\tau(i)}\right)} \cdot l\left(\alpha_{\sigma(i)}, \beta_{\sigma(i)}\right)
$$


Os coeficientes de $m(x)$ são funções simétricas de $\alpha_{1}, \alpha_{2}, \ldots, \alpha_{r}$ e $\beta_{1}, \beta_{2}, \ldots, \beta_{s}$, assim temos que estes coeficientes estão em $\mathbb{Q}$. Finalmente, $m(\theta)=m(\alpha+$ $c \beta)=l(\alpha, \beta)$, em outras palavras, $l(\alpha, \beta) \in \mathbb{Q}(\theta)$.

Agora que sabemos que toda extensão algébrica finitamente gerada é simples, podemos definir o grau de uma extensão : se $L=K(\alpha)$ e $\alpha$ é raiz de um polinômio irredutível com coeficientes em $K$ e de grau $d$, dizemos que $L$ tem grau $d$ sobre $K$ e escrevemos $d=[L: K]$. Observe que esta noção coincide com a noção usual de grau de $L$ sobre $K$, sendo $L$ um espaço vetorial sobre $K$ : basta tomar $1, \alpha, \alpha^{2}, \ldots, \alpha^{d-1}$ como base. Em particular, a noção de grau está bem definida, isto é, independe da escolha do elemento gerador de $\alpha$. Temos ainda que o grau é multiplicativo no seguinte sentido: se $E \supset L \supset K$ são corpos, então $[E: K]=[E: L][L: K]$. A demonstração é simples: se $\omega_{i}$ é uma base de $E$ sobre $L$ e $\tau_{j}$, uma base de $L$ sobre $K$, é fácil verificar que $\omega_{i} \tau_{j}$ é uma base de $E$ sobre $K$.

\section{B.4 Automorfismos e Extensões Galoisiana}

Um automorfismo de um corpo $L$ é uma imersão cuja imagem é o próprio $L$. Mais explicitamente, uma bijeção $\sigma: L \rightarrow L$ é automorfismo se $\sigma(x+y)=$ $\sigma(x)+\sigma(y)$ e $\sigma(x y)=\sigma(x) \sigma(y)$ para todo $x, y \in L$. Se $K$ é um subcorpo de $L$, diremos ainda que $\sigma$ é um automorfismo sobre $K$ se ele fixar cada elemento de $K$, isto é, se $\sigma(k)=k$ para todo $k \in K$.

É fácil ver que o conjunto de todos os automorfismos de $L$ sobre $K$ formam um grupo. Pelos resultados anteriores, temos que há no máximo $[L: K]$ tais automorfismos. Se $L=K(\alpha)$ e todos os conjugados de $\alpha$ pertencem a $L$, então haverá exatamente $[L: K]$ automorfismos, dados por $\sigma(\alpha)=\alpha^{\prime}$, em que $\alpha^{\prime}$ é um conjugado de $\alpha$. Se isto ocorrer, diremos que $L$ é uma extensão galoisiana de $K$. Neste caso, denotamos o grupo de automorfismos de $L$ sobre $K$ por $\operatorname{Gal}(L / K)$.

O jeito mais fácil (de fato, o único jeito [Jac]) de se obter uma extensão galoisiana é acrescentar a $K$ todas as raízes $\alpha_{1}, \alpha_{2}, \ldots, \alpha_{n}$ de um polinômio $p(x)$ com coeficientes em $K$; neste caso, qualquer imersão $\sigma$ de $L=$ $K\left(\alpha_{1}, \alpha_{2}, \ldots, \alpha_{n}\right)$ permuta as raízes de $p(x)$, já que $p\left(\sigma\left(\alpha_{i}\right)\right)=\sigma\left(p\left(\alpha_{i}\right)\right)=0$. Assim, $\sigma(L)=L$ e, portanto $\sigma$ é um automorfismo de $L$.

Por exemplo, se $L=\mathbb{Q}\left(x_{1}, x_{2}, \ldots, x_{n}\right)$ é o corpo das funções racionais e $K=\mathbb{Q}\left(s_{1}, s_{2}, \ldots, s_{n}\right)$, o subcorpo das funções racionais simétricas, temos que as permutações das variáveis das funções racionais definem automorfis- 
mos de $L$ sobre $K$. Além disso, como

$$
\prod_{1 \leq i \leq n}\left(x-x_{i}\right)=x^{n}-s_{1} x^{n-1}+s_{2} x^{n-2}-\cdots+(-1)^{n} s_{n}
$$

temos que $L=K\left(x_{1}, x_{2}, \ldots, x_{n}\right)$ é uma extensão galoisiana de $K$.

\section{B.5 Teorema Fundamental}

A idéia da teoria de Galois é classificar extensões de corpos através de seus grupos de automorfismos. Vejamos como.

Seja $L$ uma extensão de $K$. Podemos associar, para cada subcorpo intermediário $E$ (isto é, $L \supset E \supset K$ ), o subgrupo do grupo de automorfismos de $L$ sobre $K$ que são também automorfismos de $L$ sobre $E$, ou seja, o subgrupo dos automorfismos que fixam cada elemento de $E$. Reciprocamente, a cada subgrupo $H$ do grupo de automorfismos de $L$ sobre $K$, associamos o seu corpo fixo $L^{H}=\{x \in L \mid \sigma(x)=x \quad \forall \sigma \in H\}$.

É natural imaginar se as duas correspondências acima são inversas uma da outra, o que é verdade se exigirmos que $L$ seja extensão galoisiana de $K$. Neste caso, $L$ é uma extensão galoisiana de $E$ e temos que o grupo associado a $E$ é nada mais nada menos do que $\operatorname{Gal}(L / E)$.

Temos o seguinte

Teorema B.4 (Teorema Fundamental da Teoria de Galois) Seja L uma extensão galoisiana de $K$. Então existe uma bijeção entre os subcorpos $E$ de $L$ que contêm $K$ e os subgrupos de $\mathrm{Gal}(L / K)$, dada por

$$
E \rightarrow \operatorname{Gal}(L / E)
$$

e cuja inversa é

$$
H \rightarrow L^{H}
$$

Demonstração Sejam $E$ um subcorpo de $L$ e $H=\operatorname{Gal}(L / E)$. Pela definição, sabemos que $L^{H} \supset E$. Para mostrar a inclusão reversa, escrevemos $L=E(\theta)$. Seja $p(x)$ o polinômio minimal de $\theta$ sobre $E$ e sejam $\theta_{1}=\theta, \theta_{2}, \ldots, \theta_{r}$ as raízes conjugadas de $\theta$, que estão em $L$ pois $L$ é uma extensão galoisiana de $E$. Temos então que os automorfismos de $H$ são dados 
por $\sigma_{i}(\theta)=\theta_{i}, i=1,2, \ldots, r$. Agora, seja $l \in L^{H}$, então podemos escrever $l=l(\theta)$ para alguma função racional de $\theta$ com coeficientes em $E$ e

$$
l=\frac{1}{r} \sum_{\sigma \in H} \sigma(l)=\frac{l\left(\theta_{1}\right)+l\left(\theta_{2}\right)+\cdots+l\left(\theta_{r}\right)}{r},
$$

que é uma expressão simétrica das raízes de $p(x)$, logo é um elemento de $E$. Portanto $L^{H} \subset E$ e, assim, $L^{H}=E$.

Para finalizar, temos que se $H$ é um subgrupo de $\operatorname{Gal}(L / K)$ e $E=L^{H}$, então $H \subset \operatorname{Gal}(L / E)$. Novamente, escrevemos $L=E(\theta)$ e consideramos o polinômio

$$
p(x)=\prod_{\sigma \in H}(x-\sigma(\theta))
$$

Temos os coeficientes de $p(x)$ são fixados por elementos de $H$, logo pertencem a $E$. Assim, $|\operatorname{Gal}(L / E)|=[L: E] \leq|H|$. Da inclusão anterior, concluímos que $H=\operatorname{Gal}(L / E)$. 


\section{Referências Bibliográficas}

[Arv] Arvind, V., Beigel, R., Lozano, A., The Complexity of Modular Graph Automorphism, SIAM J. Comput. 30, no. 4, 2000.

[Bab] Babai, László, Automorphism Groups, Isomorphism, Reconstruction, Handbook of Combinatorics, vol. II, Elsevier Science B. V., 1995.

[Coh] Cohen, Henri, A Course in Computational Algebraic Number Theory, Graduate Texts in Mathematics 138, Springer-Verlag, 1995.

[Had] Hadlock, Charles R., Field Theory and its Classical Problems, Carus Mathematical Monographs 19, MAA, 1978.

[Hal] Hall Jr., Marshall, The Theory of Groups, Second Edition, Chelsea Publishing Company, 1976.

[Hof] Hoffmann, Christoph M., Group-Theoretic Algorithms and Graph Isomorphism, Lecture Notes in Computer Science 136, Springer-Verlag, 1982.

[Jac] Jacobson, Nathan, Basic Algebra I, Second Edition, W. H. Freeman and Company, 1985.

[Luk] Luks, Eugene M., Isomorphism of Graphs of Bounded Valence Can Be Tested in Polynomial Time, Journal of Computer and System Sciences 25, 42-65, Academic Press, 1982.

[McK] McKay, Brendan D., Nauty, http://cs .anu.edu.au/bdm/nauty. 


\section{Índice Remissivo}

algoritmo

de Luks, 35

de Sims, 20

anel local, 62

automorfismo

de Frobenius, 58

de grafos, 7

de uma extensão, 68

sobre um corpo, 68

cadeia de estabilizadores, 18

ciclo, 15

classe $\Gamma_{k}, 32$

corpo

das funções racionais, 68

das funções racionais simétricas, 68

fixo, 69

especialização, 50

estabilizador de um elemento, 17

extensão

galoisiana, 68

grau de, 68

filtragem, 19

grupo

conjunto de geradores, 16

das transformações lineares inversíveis, 41 das transformações afins, 41

de Galois, 68

diedral, 16

grupo de permutações, 16

ação, 16

imprimitivo, 24

grau de, 16

órbita, 17

primitivo, 24

transitivo, 17

ideal primo, 62

imersão, 65

isomorfismo

de grafos rotulados, 9

de grafos, 7

localização, 62

ordem grau-lexicográfica, 64

$p$-filtragem, 23

polinômio

simétrico elementar, 63

minimal, 66

simétrico, 63

produto trançado (wreath product),

47

raízes conjugadas, 66

representação, 16

regular, 16 
fiel, 16

regular associada a um subgrupo, 17

série de composição, 32

sistema de imprimitividade, 24

socle, 38

teorema de Cayley, 16

termo líder, 64 